Invited paper submitted to the Journal of Intelligence

\title{
MIND-PERSONALITY RELATIONS FROM CHILDHOOD TO EARLY ADULTHOOD
}

\author{
Andreas Demetriou ${ }^{1}$, George Spanoudis ${ }^{2}$, Mislav Zebec ${ }^{3}$, \\ Maria Andreou ${ }^{1}$, Hudson Golino ${ }^{4}$, and Smaragda Kazi ${ }^{5}$ \\ 1. University of Nicosia, Cyprus \\ 2. University of Cyprus, Cyprus \\ 3. University of Zagreb, Croatia \\ 4. University of Virginia, USA
}

5. Panteion University of Social Sciences, Greece

Address: University of Nicosia, Makedonitissas Avenue, P.O. Box 24005, 1700 Nicosia, Cyprus Email: ademetriou@ucy.ac.cy 


\begin{abstract}
We present three studies which investigated the relations between cognition and personality from 7 to 20 years of age. The first investigated developmental relations between three reasoning domains (inductive, deductive, and scientific) and Eysenck's four personality dimensions in a design where 260 9-14-year-olds received the cognitive tests 3 and the personality test 2 times. It was found that initial social likeability and extroversion significantly shape developmental momentum in cognition and vice-versa, especially in the 9 to 11 years period. The second study involved 438 participants from 7 to 17 years, tested twice on attention control, working memory, reasoning in different domains, and once by a Big Five Factors inventory. Extending the findings of the first, this study showed that progression in reasoning is affected negatively by conscientiousness and positively by openness, on top of attention control and working memory influences. The third study tested the relations between reasoning in several domains, the ability to evaluate one's own cognitive performance, selfrepresentation about the reasoning, the Big Five, and several aspects of emotional intelligence, from 9 to 20 years of age $(\mathrm{N}=247)$. Network, Hierarchical Network, and Structural Equation modeling showed that cognition and personality are distinct but related, interfaced by the ability of self-knowing. Emotional intelligence was not an autonomous dimension. All dimensions but emotional intelligence influenced academic performance. A developmental model for mind-personality relations is proposed.
\end{abstract}




\section{MIND-PERSONALITY RELATIONS \\ FROM CHILDHOOD TO EARLY ADULTHOOD}

Mental processes and motivational and personality dispositions interact to guide understanding and action in the world. Wechsler argued that "general intelligence cannot be equated with intellectual ability but must be regarded as a manifestation of the personality as a whole." (Wechsler, 1950, p. 83). Along this line, Allport (1937) suggested that personality is "the dynamic organization within the individual of those psychophysical systems that determine his unique adjustment to his environment" (p. 48). In Cattell's (1965) model intelligence and personality occupy equal standing. However, there is no generally acceptable model specifying when and how cognitive and personality processes interact. Recognizing this lack, Jensen (1998) concluded “The g factor", his magnum opus on general intelligence, urging researchers to study the intelligence-personality nexus.

The studies presented here explore this nexus, tracing its changes in development. It is well known that, on the one hand, individuals at different ages understand the world differently despite possible similarities in personality (Demetriou \& Spanoudis, 2018); on the other hand, early differences in personality may channel individuals to relate differently with the world, despite their intellectual similarities, eventually differentiating their developmental opportunities (Caspi, 2005; Rothbart, 2011). For instance, reflective individuals may control motivational and personality dispositions, achieving better adaptation in their environment than less reflective individuals. No satisfactory model exists for these interactions. The research presented here aspires to lay the ground for this model. We present three studies related to the focus of this special issue. Specifically, we examine how personality and 
cognitive processes interact from childhood to early adulthood, mapping how their relations change with development. Also, we present research on the relative contribution of each of these factors to real life achievements, such as academic achievement. Below we review literature related to the organization of intelligence and personality, their development, and their possible relations in development. This introduction will conclude with predictions suggested by this research.

\section{The Organization of Cognition and Personality: Commonalities and Differences}

Cognition. In the recent years, there has been significant progress in the study of the organization of intelligence and personality and their possible common ground (e.g., Ackerman, 2018; Chamorro-Premuzic \& Furnham, 2006; Ziegler, Danay, Heene, Asendorpf, \& Bühner, 2012). There is consensus that both are hierarchically organized. Specifically, intelligence is a three-level hierarchical structure involving three types of systems: (i) several broad domains of ability, such as spatial, verbal, and quantitative reasoning; (ii) domaingeneral systems of inferential and representational processes, such as fluid intelligence (Gf; basically inductive reasoning allowing processing of similarities and relations at increasing levels of abstraction) and crystallized intelligence (Gc; knowledge and skill systems crystallizing learning and experience that may be activated for understanding and problem solving; (ii) a general factor (g) mainly associated with processing efficiency, attention control, mental flexibility, and working memory (Carroll, 1993; Flynn, 2009; Jensen, 1998; Hunt, 2011). Cognizance was recently added as a major background process of $\mathrm{g}$. This includes self-monitoring of mental and behavioral processes, representation and awareness of them, and systematic modification of them, including metarepresentation which generates new 
mental and behavioral constructs (Demetriou \& Spanoudis, 2018, Demetriou et al., 2017, 2018a, 2018b).

Personality. In personality, a structurally equivalent hierarchical model is gaining popularity. This model specifies several dimensions standing for specific dispositions to relate with the world. According to the Big Five Factors model, which dominates the field, these dimensions are: extraversion (E, enjoying being with others and actively seeking social company and activity); agreeableness (A, oriented to others, trust them and be warm with and make good to them); conscientiousness ( $\mathrm{C}$, goal-minded, focused, careful, organized, determined, and planful, neuroticism $(\mathrm{N}$, individuals high in neuroticism are disturbed by variations in the environment so that they are nervous, anxious, and moody); openness to experience/intellect $(\mathrm{O}$, curious, try new experiences, inventive, original, and imaginative with wide interests). The Big Five Model integrated constructs from earlier models of personality. It shares two of the Big Five with Eysenck's (Eysenck \& Eysenck, 1969) theory (E and N) and overlaps with the third, Psychoticism (P), which combines traits of A and C (Eysenck, 1992a, 1992b; Costa \& McCrae, 1992). It also integrated openness from Cattell' (1965) model, standing for the projection of mental ability in personality.

Evidence shows that three of the Big Five Factors relate to one and the rest to another second-order factor: $\mathrm{C}, \mathrm{N}$, and A relate to the general trait of stability, the $\alpha$-factor, underlying efficiency in organizing one's own life, dealing with pressure, and making oneself acceptable; the other, the beta factor ( $\beta$-factor), relates with $\mathrm{O}$ and $\mathrm{E}$, and stands for plasticity in one's relation with the world. In turn, these two factors relate to a third-order general factor, the General factor of Personality (GFP). Broadly speaking, these factors appear related to the higher-order factors in the hierarchical model of intelligence outlined above. Stability 
associates with crystallized intelligence, including dispositions and skills underlying interactions with the social world; plasticity expresses fluid intelligence in personality. "The GFP is analogous to $g$ and predicts social efficiency in the way g predicts cognitive efficiency" (Rushton \& Irwing, 2009, p. 564). GFP, like g, relates to actual life indicators, such as performance at school and work (e.g., van der Linden, te Nijenhuis, \& Bakker, 2010).

Despite their possible similarities, there is a large uncharted territory between intelligence and personality. For instance, the second-order $\alpha$ - and $\beta$-factors in personality organize different first-order factors. This is not the case in intelligence: $\mathrm{Gf}$ and Gc stand for different components involved in each one of first-order factor rather than different factors. Also, it is not clear how some common background processes operate in personality and intelligence. Executive control, the ability to focus on stimuli, plan action, and flexibly change focus as needed, is a strong example. In current models of intelligence, it is a central component of Gf (Blair, 2006; Demetriou et al., 2017, 2018a, 2018b). In personality, it may be involved in two different, often orthogonal, dimensions. As a mechanism of regulation and planning, executive control (MacDonald, 2008) may underlie the self-discipline and stability involved in C; as a mechanism of flexibility, it may be a key mechanism for sustaining plasticity in dealing with others (E) or with novelty (O) (Rothbart, 2011). We know of no research that explored these questions. Also, all personality theories, including the Big Five Factor model, are largely based on self-rating inventories. Therefore, the various dimensions of personality may to a large extent reflect self-representations rather than actual behaviors or actual dispositions. Notably, there is strong evidence that GFP is highly self-representational, reflecting a person's self-concept and self-worth rather than actual cognitive ability: the relation between GFP and self-esteem is very high (67\% of the variance) (Erdle, Irwing, 
Rushton, \& Park, 2010). Also, self-represented cognitive g accounts for much more of the variance of all Big Five (between 18-25\%) but N (1\%) than Gf (between 1-3\%) (Demetriou, Kyriakides, \&Avraamidou, 2003).

\section{Development of Cognition and Personality}

Cognitive development. There is general agreement that intelligence develops over a series of levels from birth to early adulthood. Macroscopically, theories of cognitive development agree that major transitions occur around the age of 2, 7, and 11 years, when thought changes in abstraction, cohesion, and reasoning power. These changes were associated with increasing processing and representational efficiency as indicated by functions such as processing speed, attention control, and working memory (Case, 1985; Pascual-Leone, 1987; Piaget, 1970). Integrating over these theories and a long series of empirical studies, we postulated that cognitive development occurs in four major developmental cycles, with two phases in each. New representations emerge in the first phase of each cycle and their integration dominates in the second. Transitions across cycles is driven by increasing mental self-awareness and self-regulation (Demetriou \& Efklides, 2018; Demetriou et al, 2018b).

In succession, the four cycles operate with episodic representations from birth to 2 years, realistic mental representations from 2 to 6 years, generic rules organizing representations into conceptual/action systems from 6 to 11 years, and overarching principles integrating rules into systems where truth and multiple relations can be evaluated from 11 to 18 years. Episodic representations are remembrances of actions and experiences preserving their spatial and time properties. Realistic representations are blueprints of episodic representations where spatial and time properties are reduced, associated with personal 
symbols, such as mental images, or conventional symbols, such as words. Rules are abstract mental schemes capturing relations between realistic representations and expressing them as concepts or categories of things, causal relations, etc. Principles are higher-order rules specifying how generic rules are related or how they may be integrated. Changes within cycles occur at about their middle, at 4, 8, and 14 years, when representations become explicitly cognized so that their relations can be worked out, gradually resulting into representations of the next cycle. Therefore, these cycles are distinguished from each other by the type of representation dominating in each (i.e., episodic schemes, mentations, rules, and principles) and the relations connecting representations (i.e., spatially and time-based associations, representational mappings, inferential links, truth- or validity-based inferential constraints).

Cognizance is central in this developmental system. It defines the subjective aspect of mental functioning, raising it from simple computation to representation where information and mental functioning is subjectively meaningful. Thus, cognizance is a major source of transitions across cycles. The self-representation system gradually builds pointers to different combinations of (i) problem solving skills and processes, (ii) dispositions to go on with a particular pattern of activity or abandon it, and (iii) feedback received about successes and failures and the ensuing feelings of satisfaction and dissatisfaction. These pointers are used by the person for self-regulation and self-representation, enabling him or her to choose appropriate action patterns among alternatives. Thus, both action patterns and selfrepresentations come out as packages involving combinations of abilities, dispositions, styles, and interests. Cognizance is cycle-specific, exerted through the representational processes dominating in each cycle: it is based on perception- and iconic-like representations in the representational cycle; rule-based inferential processes in the rule-based cycle; and abstract 
semantic processes in the principle-based cycle. It becomes increasingly accurate along the cycles resulting into increasingly refined understanding of the functioning of the mind and increasingly accurate self-representations, specifying personal strengths and weakness.

Personality development. Precursors of adult personality dimensions are established very early in life. Temperament, which reflects differences between children in their reactivity to external stimuli and their ability for self-regulation are present since infancy (Rothbart, 2011). The Big Five Factors are discernible from early childhood; however, in line with our predictions above, their reliability and stability increase with age (Asendorph \& van Aken, 2003; Lamb, Chuang, Wessles, Broberg, \& Hwang, 2002). Overall, conscientiousness, irritability, and positive activity were present and relatively stable since early childhood; extraversion and neuroticism stabilized after the age of 8 years; openness was never stable, suggesting that it may not be a meaningful dimension of personality prior to adolescence. Also, the prevalence of various factors varies with age: children become less extroverted but more agreeable, conscientious, and emotionally stable as they grow; openness increases in childhood and adolescence and decreases later (Lamb et al., 2002; McCrae et al., 2000; Roberts, Walton, \& Viechtbauer, 2006).

\section{Predictions: Interactions between Intelligence and Personality}

The relation between some aspects of intelligence and some aspects of personality is better established than between others. For instance, the positive relation between fluid and crystallized intelligence and openness is well established (Ackerman, 2018). In development, Ziegler et al (2012) showed longitudinally that openness positively influences the development of fluid intelligence from adolescence to early adulthood. Also, Antinori, Carter, and Smillie (2017) showed that open individuals 'see' more possibilities in the input and they flexibly 
combine information from the two eyes in a creative fashion, especially under a positive mood. Along this line, personality differences influence selective attention to stimuli. McIntyre and Graziano (2016) showed that individuals oriented to other persons tend to selectively attend to social stimuli; individuals oriented to inanimate things tend to selectively attend to objects.

Based on the literature above, the studies presented here are relevant to the following predictions. First, at a general level, self-representations of any characteristic become increasingly accurate with age. Normally, self-ratings expressing self-representations, which is typical in personality research, may be relatively valid with late rule-based thought or later. Self-ratings require (i) relatively explicit self-representations that may be subjectively dimensionalized and (ii) mapping these subjective dimensions onto a scale varying along a certain metric specified by the researcher. Both abilities are not present before late rule-based thought. In fact, it is only with principle-based thought, in adolescence, that persons possess an elaborate self-evaluation ability and a refined self-concept that they may use to specify their cognitive, emotional, personality, and behavioral characteristics (Demetriou, 2000). Therefore, progression along the cognitive cycles above would be reflected in personality ratings, tuning them with cognized changes in mental possibilities and related outcomes, such as learning. At the very least, the self-ratings may change in time to reflect cognitive changes in thought complexity and resolution of self-monitoring and self-representation.

Second, given the self-representational nature of personality evaluation, cognizance would relate with personality more than procedural aspects of intelligence, such as processing efficiency or Gf. Moreover, changes in cognitive processes would relate more with aspects of personality reflecting a personal value-laden judgement of oneself, such as Eysenck's social 
likeability, than with aspects standing for stable personal dispositions, such as neuroticism. These differential relations would reflect influences of cognitive change on the fine-tuning of one's self-representation and self-worth vis-à-vis the world.

Third, this differential relation between cognitive development and personality would occur from the point of view of personality as well. Overall, (i) there is clear evidence that some personality dimensions (i.e., $\mathrm{O}$ and $\mathrm{C}$ ) are more related to intelligence than others (i.e., $\mathrm{P}$, $\mathrm{N}$, and A). This pattern suggests that the first group would relate to cognitive development more than the second group. However, (ii) even the dimensions in the first group would differentially relate with change. There is an apparent paradox in the relations of $\mathrm{C}$ with intelligence and academic performance: $\mathrm{C}$ is negatively related to intelligence but positively with academic performance (Chamorro-Premuzic \& Furnham, 2006; Demetriou et al., 2003).

\section{Study 1: Developmental Changes in Personality-Intelligence Relations}

The present study was part of a longitudinal study of cognitive development covering the age span from 9 to 18 years of age (see Demetriou, Christou, Spanoudis, \& Platsidou, 2002). The study focused on the development of inductive (fluid) reasoning, deductive syllogistic reasoning, and scientific reasoning underlying the ability to test hypotheses by specifically designed experiments. Participants were tested three times separated by 1-year intervals by a specifically designed Raven-like Matrices test, a test of syllogistic reasoning addressed to various logical schemes and a test of scientific reasoning requiring isolation of variables in designing experiments at several levels of complexity (Demetriou \& Kyriakides, 2006). At the first two testing waves participants were also tested by the Eysenck Personality Questionnaire (EPQ) (Barrett, Petrides, Eysenck, \& Eysenck, 1998). Therefore, this study allows testing longitudinally 
how cognitive changes relate with possible personality changes in the transition from childhood to adolescence.

Eysenck's theory of personality specified four factors: extraversion $(\mathrm{E})$, neuroticism $(\mathrm{N})$, psychoticism (P), and social likeability (L). We noted above that two of these factors, E and N, are by and large the same with the corresponding factors in the Big Five Factor model. There is less agreement about the rest two. Specifically, $\mathrm{P}$ is characterized by aggression, assertiveness, egocentric and manipulative behaviors, orientation to achievement, dogmatism, and toughmindedness. Notably Eysenck (1992) himself and Costa \& McCrae (1992), the proponents of the Big Five Factor model, agree that $\mathrm{P}$ involves a combination of traits of $\mathrm{A}$ and $\mathrm{C}$. Empirical evidence did show that $\mathrm{P}$ involves $\mathrm{C}$ and Impulsive Sensation Seeking, indicating lack of planning and a tendency to act without thinking (Zuckerman, Kuhlman, Joireman, Teta, \& Kraft, 1993). Individuals high in $L$ tend to give positive characteristics to themselves in their relations with others: i.e., that they are considerate, good mannered, and faithful to others, own enjoying being with and co-operating with the, follow the rules, and recognize their mistakes. This scale correlates with Impulsive Sensation Seeking (Zuckerman et al., 1993).

Customizing the general predictions stated above on the present study three specific predictions may be tested. First, changes in both cognition and personality in the earlier part of the age period examined here would be larger than in the later part to signify the transition from rule- to principle-based thought from childhood to adolescence. Second, a privileged relation of L to cognitive change relative to the other personality dimensions is also to be expected: this dimension reflects the accuracy of self-evaluations more than the other dimensions because it activates one's wish to be perceived positively by others. Finally, E may also be preferentially linked to cognitive change, as the representative of cognitive flexibility in this study. 


\section{Method}

\section{Participants}

A total of 260 participants were examined, longitudinally covering the age span from nine to 16 years. Specifically, this total included $444^{\text {th }}$ grade primary-school children (mean age 9.6 years old at first testing; 31 males), and 46 (mean age 12.6 years at first testing; 21 males), 92 (mean age 13.6 years at first testing; 46 males), and 78 (mean age 14.6 years at first testing, 31 males) $1^{\text {st }}, 2^{\text {nd }}$, and $3^{\text {rd }}$ grade secondary school students, respectively. All cohorts were tested three times by the cognitive battery and two times by the EPQ (in March or April) separated by one-year intervals. Participants came from middle or high SES families with parents with university education.

\section{Task Batteries}

The fluid reasoning battery included 12 Raven-like matrices specifically designed to address the following four levels of complexity: (i) integration of two attributes varying in the same direction (e.g., geometric figures of increasing size and with a background mark increasing in the same direction; (ii) integration of intersecting elements, such as size and shape, marked elements such as dots at a particular position, without transformations; (iii) dissociation of relevant from irrelevant elements or simple transformations such as the rotation of a line across matrices; and (iv) integration of multiple properties varying according to several rules (e.g., change in shape, size and position of some attribute).

The deductive reasoning battery include 20 items addressed to different types of logical relations scaling in four levels: (i) Modus ponens (implication) and disjunctive reasoning; (ii) modus tolens and falsification of disjunctive propositions; (iii) understanding of argument of falsification and grasp of non-decidability of arguments; (iv) explicit grasp of the rules of 
implicative reasoning and full mastery of fallacies (affirmation of the consequent and denial of the antecedent).

The scientific reasoning battery included 16 items addressed to the following four levels: (i) identification of confounding variables and interpretation of the results of simple experiments (matching variables with their effects); (ii) systematic isolation of variables of explicit hypotheses; (iii) design experiments verifying a hypothesis and interpretation of evidence suggesting various causal relations (necessary and non-sufficient; neither necessary nor sufficient; incompatible); (iv) design experiments (i) to falsify a hypothesis, (ii) verify each of the above causal relations, and model construction.

The full version of the Eysenck Personality Questionnaire (EPQ) was used. EPQ involves 80 items addressed, 20 for each four personality dimensions specified in Eysenck's theory (Eysenck \& Eysenck, 1969).

Scoring

All items in the cognitive batteries were scored on a pass-fail basis. To pass a matrix, two empty cells (the second and third of the second and third raw, respectively) would have to be filled in by choosing the proper options among 8 alternatives. To pass a syllogism, the logically proper answer would have to be selected among three alternatives. To pass a scientific reasoning item answers would have to indicate task-relevant control of variables, hypothesis formation, or data interpretation.

A level score was given to each participant for each battery; this is the highest level at which a person solved two thirds or more of the tasks at this level. The level structure of each battery was validated and fine-tuned by means of Discrimination Level Analysis. This method was developed by Shayer to score individual performance on batteries involving 
developmentally scaled tasks (Shayer, Demetriou, \& Prevez, 1988). This method showed that the four-level sequence proved very consistent for each battery. In concern to the developmental cycles summarized in the introduction, the first two levels of the inductive and deductive reasoning batteries addressed early and late rule-based thought and the last two address principle-based thought. Level I of the scientific reasoning battery addressed late rule-based thought; level II addressed early principle-based thought; levels III and IV addressed lateprinciple-based thought. Thus, a level score, ranging from 0 (not satisfying ascription to the lower level of a scale) to 4 was ascribed to each person for each battery at each testing wave. These scores were used in the various analyses to be presented below. All three batteries were very reliable (Crombach's alpha always $>.8$; mean inter-rater agreement for the scientific reasoning battery was $85.2 \%, \mathrm{sd}=9.7$ ). Within battery correlations of level scores across waves were high (.45-.67); correlations across batteries were lower but still high (.29-.56) to

Responses to the items in the EPQ were identified as yes/no (applies vs. does not apply to me) and the participant's score on each dimension was the sum of the items judged to apply. Test-retest reliability was high (.77). Self-correlations across waves were very high: .72-.85; $\mathrm{N}$ and $\mathrm{P}$ correlated positively with each other within and across waves (.18 - .22); E correlated positively with $\mathrm{P}(.11-.13)$ and negatively with $\mathrm{N}(-.13--.15)$; L correlated highly and negatively with $\mathrm{P}$ and $\mathrm{N}(-.39--.49)$ and negatively but low with $\mathrm{E}(-.05--.09)$. Correlations of three personality dimensions with the three cognitive scales were low to moderate (.00- .26$)$; the correlations of $\mathrm{L}$ with the three cognitive dimensions were much higher, but always negative $(-.22--.45)$, suggesting a systematic developmental relation.

\section{Results}


The pattern of performance on the cognitive tests is shown in Figure 1. The effect of age, $\mathrm{F}(3,252)=120.415, \mathrm{p}<.0001$, partial $\eta^{2}=.59$, was highly significant and very powerful, reflecting large improvement of performance across age groups. The effect of testing wave was also significant, $\mathrm{F}(2,251)=238.813, \mathrm{p}<.0001$, partial $\eta^{2}=.66$, reflecting large improvement across testing waves. The effect of cognitive domain was also highly significant, $\mathrm{F}(3,251)=$ $259.668, \mathrm{p}<.0001$, partial $\eta^{2}=.67$, reflecting variation of performance across domains:

performance on inductive reasoning was higher than on deductive reasoning and this was higher than scientific reasoning. The interactions between age and wave $\mathrm{F}(6,504)=7.732, \mathrm{p}<.0001$, partial $\eta^{2}=.08$, and wave and domain, $F(4,249)=7.756, p<.0001$, partial $\eta^{2}=.11$, were also significant, indicating two trends: first, the magnitude of improvement across waves varied with age; it was much larger at lower ages, indicating transition from rule-to principle-based thought and approaching ceiling at older ages; second change was almost linear across domains in inductive and deductive reasoning but larger from first to second than from second to third in scientific reasoning. Noticeably, no effect of gender ever reached significance. 


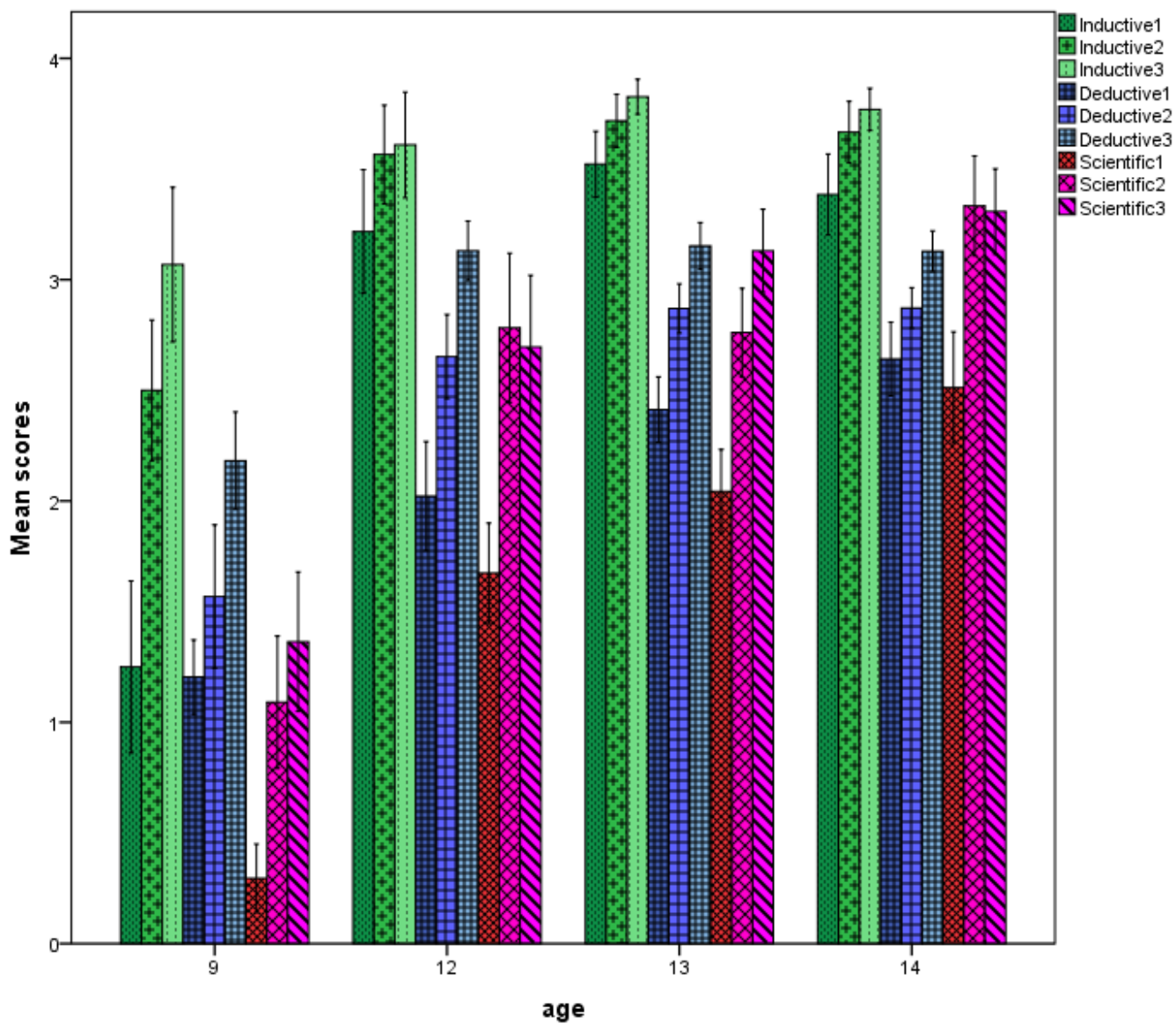

Figure 1. Performance on the three cognitive batteries across age and testing waves.

There were similarities and differences in the pattern of relations between age, testing wave, and personality dimensions. Specifically, the effect of age, $F(3,256)=9.307, p<.0001$, partial $\eta^{2}=.10$; wave, $F(1,256)=15.390, \mathrm{p}<.0001$, partial $\eta^{2}=.06$, and dimension was significant, $\mathrm{F}(3,254)=1811.559, \mathrm{p}<.0001$, partial $\eta^{2}=.96$. Also, the age by dimension, $\mathrm{F}(9$, $768)=8.264, \mathrm{p}<.0001$, partial $\eta^{2}=.09$, and the waves by dimension interaction was highly significant, $\mathrm{F}(3,256)=28.287, \mathrm{p}<.0001$, partial $\eta^{2}=.26$ indicating that the relation with age differed across dimensions. Figure 2 illustrates these effects: $\mathrm{P}$ and $\mathrm{N}$ increased across the first three age groups and decreased at the last age group; scores in E increased from 9 to 11 years, decreased from 11 to 13 and then increased again; scores in L decreased across the first three age 
groups and stabilized there. Across waves, $\mathrm{P}$ and $\mathrm{E}$ increased; $\mathrm{N}$ and $\mathrm{L}$ decreased. Thus, in line with our first prediction, patterns of change in personality were larger earlier in age, largely reflecting patterns of cognitive change.

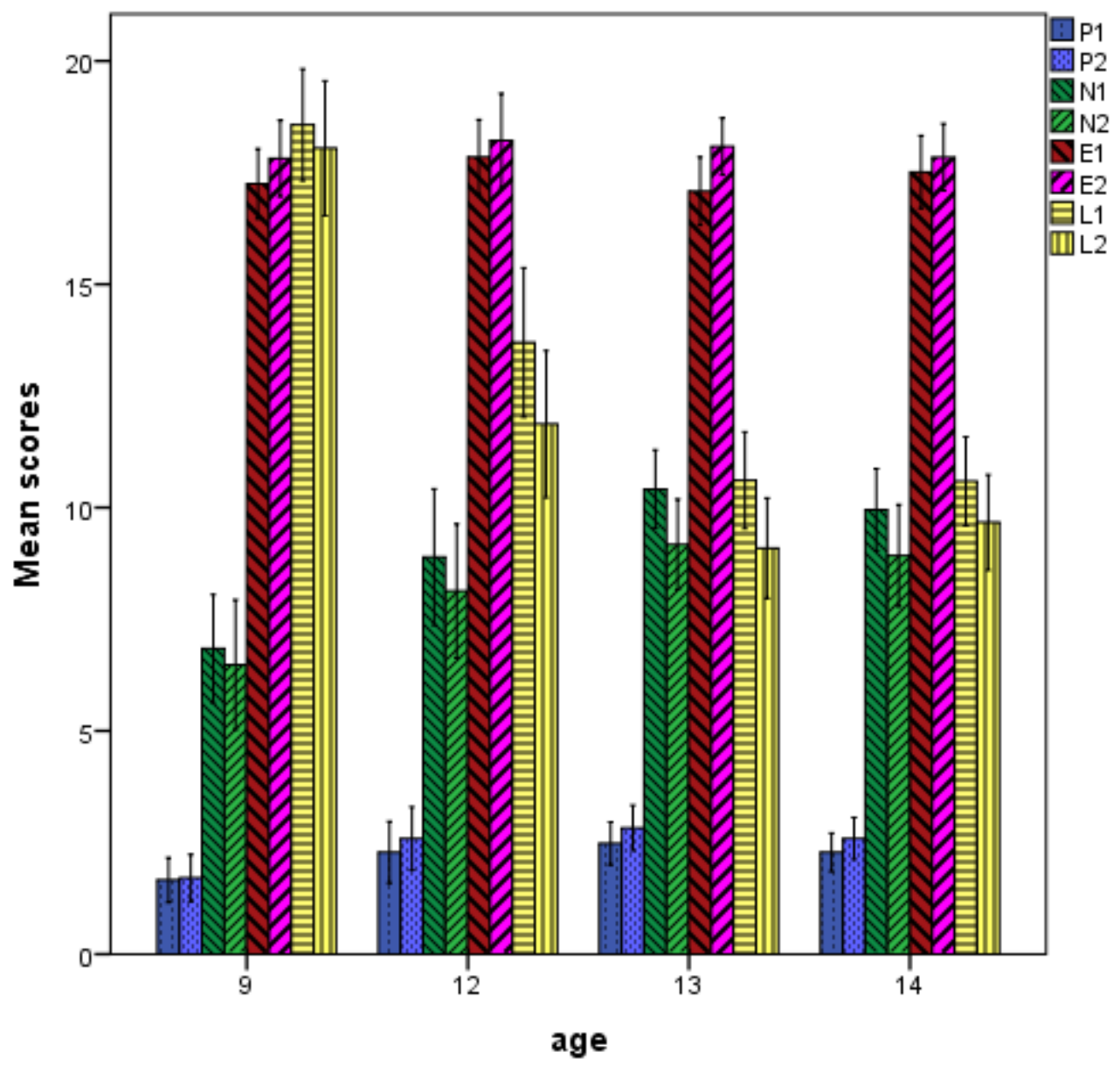

Figure 2. Personality scores across age, testing wave, and personality dimensions

\section{Cognition-Personality Relations with Development}

Two approaches were adopted to specify the relations between changes in mental and personality processes. First, a cross-lagged model of the relations between cognition and personality relations was tested to specify possible exchange of influences with the progression 
of time. This model involved the following factors: one factor, related to all three reasoning domains, for cognitive processes at each testing wave; this factor stands for Gf. There were three personality factors for each testing wave: an emotional stability factor related to $\mathrm{P}$ and $\mathrm{N}$; $\mathrm{E}$ was related to one factor and $\mathrm{L}$ to another. To specify the cross-lagged relation between factors, each factor at the second wave was related to all factors of the first wave and each factor at the third wave was related to all factors at the second wave. Figure 3 shows the model after dropping nonsignificant relations, $\left.\chi^{2}(107)=271.84, \mathrm{CFI}=1.0, \mathrm{RMSEA}=.078\right)$. Naturally, self-relations across time were very high both for the cognitive $(>.9)$ and personality factors $(>.8)$. Although small, there were cross-lagged relations between most factors. Attention is drawn to the pivotal role of L. Specifically, the effect of L at first testing on Gf (.14), E (.16) and emotional stability (-.16) at second testing was significant; the effect of Gf at first testing on $\mathrm{L}$ at second testing (.16) and $\mathrm{E}$ at first testing on Gf at third testing (.11) was significant. All but one of the between factor correlations at first testing were significant. Therefore, cognitive and personality, however weakly, do interact systematically during development so that changes in each are related to changes in the other.

The second model aimed to further specify these interactions. This was a growth model which examined the possible influence of personality on the form of Gf development in the three years covered by this longitudinal study. In sake of this aim, all three cognitive scores of each testing wave were related to a wave-specific Gf factor. The relation between these three factors and the intercept was constrained to unity to capture the initial mean of the growth function. To specify the degree of change across the three testing waves, the relation between the three wavespecific factors and slope was constrained to 0,1 , and 2 , respectively. To specify the possible distinct influence of personality on the intercept and slope, $\mathrm{P}, \mathrm{N}$, and $\mathrm{E}$ were regressed on $\mathrm{L}$. The 


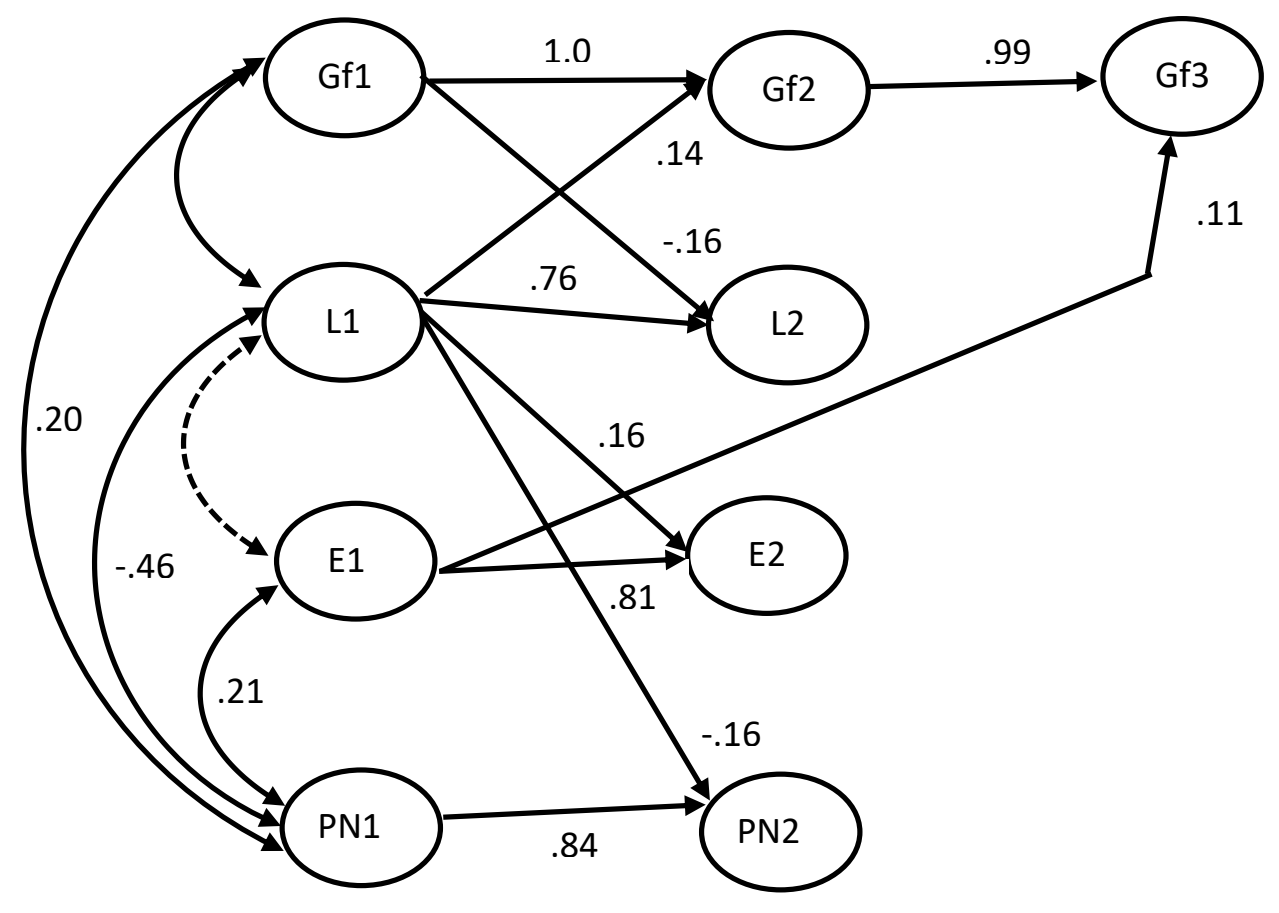

Figure 3. Cross-lagged model of the relations between Gf and personality factors across testing waves.

$\chi^{2}(107)=271.84, \mathrm{CFI}=1.00, \mathrm{RMSEA}=.078$.

intercept and slope of Gf were regressed on $\mathrm{L}$ and the residuals of each of the other three personality factors. This manipulation allows to distinctly specify the effects of each personality factor on the two Gf growth parameters. The fit of this model was very good, $\chi^{2}$ $(13)=17.02, p>.20$, RMSEA $=.065$. This model is shown in Figure 4. It may be seen that the intercept was negatively and highly related to the L factor (-.68) and moderately but significantly to the $\mathrm{P}(-.29)$ and the $\mathrm{N}(-.21)$. These effects suggest that initial high scores in $\mathrm{L}$ 
but also in factors reflecting low emotional stability are associated with comparatively lower cognitive performance.

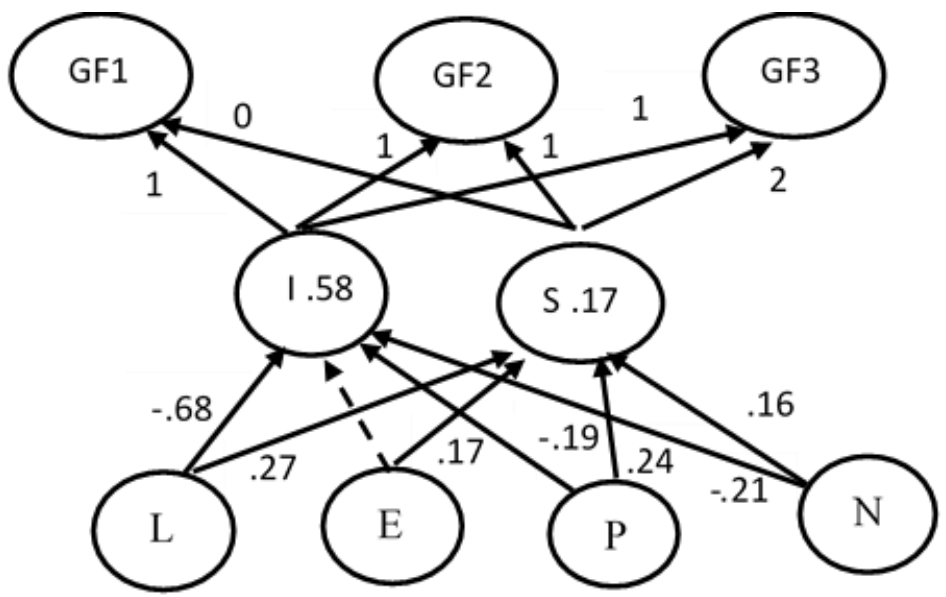

$\mathrm{X}^{2}(13)=17.02, \mathrm{p}>.20, \mathrm{CFI}=.997, \mathrm{RMSEA}=.065(.052-.06), \mathrm{AIC}=-404.51$.

Figure 4. The growth model of Gf with personality effect on Gf intercept and slope.

However, the relation of personality factors with slope was significant and positive (.27, .17, .24 for $\mathrm{L}, \mathrm{E}$, and $\mathrm{P}$, respectively). This implies that initial high scores in these factors were 
associated with larger cognitive change.

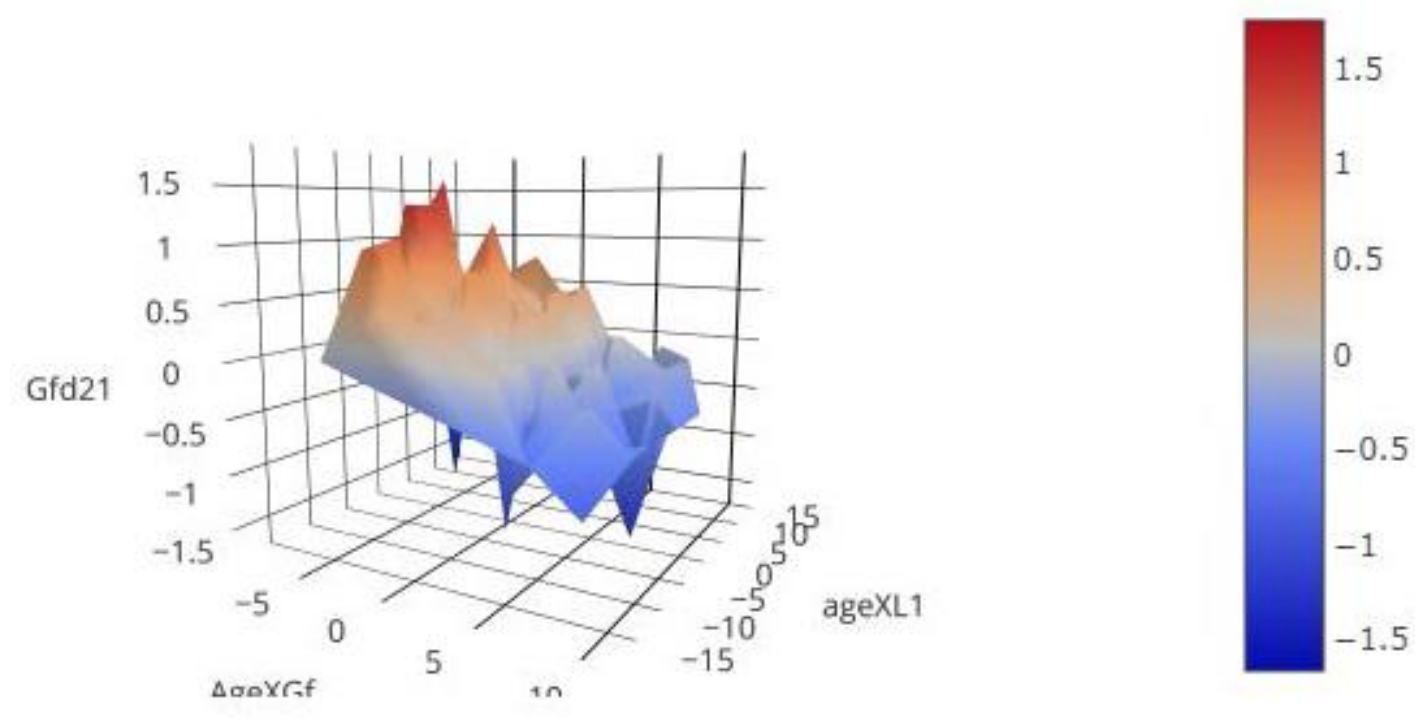

Figure 5. Relations between Gf and $\mathrm{L}\left(\mathrm{R}^{2}=.36\right)$ and change in Gf from first to second testing wave with $\mathrm{L}\left(\mathrm{R}^{2}=.05\right)$.

To further specify these relations, Latent Transition Analysis (LTA) was employed (Muthen \& Asparouhov, 2011). LTA specifies how individuals move across categories in a period of interest and the factors possibly affecting this movement. Here the first two waves were involved. Two categories were specified, one for cognitive performance at first wave and one for cognitive performance at second wave. The level score on each of the three cognitive abilities at each testing wave were used. There were two classes of participants in each category: those changing into a higher level and those staying on or regressing from their initial level. The mean score on the two emotional stability factors (P and N), E, and L were involved as covariates to examine how personality factors affect transition to a higher level 
across the first two testing. Practically, we regressed transition to a higher level across the two waves on these three personality scores to examine how they influence the likelihood of change.

This model accounted for the patterns of performance observed well (Pearson ChiSquare $(15528)=2168.783, \mathrm{p}=1.0 ;$ Likelihood Ratio Chi-Square $(15528)=730.633, \mathrm{p}=1.0$; Entropy $=.92)$. The probability of moving to a higher level was higher $(.56)$ than staying to the same level (.44) (odds to progress was 1.27). Of the various covariates, only L exerted a significant influence on transition $(1.58, \mathrm{p}<.0001$; odds 4.83). Overall then, higher scores in $\mathrm{L}$ at the start were associated with transition to a higher cognitive level. It is noted, however, that despite this overall relation, the cubic relation between cognitive attainment and the product of age by social likeability suggest that early in age (from late childhood to adolescence) it is more likely to change when $\mathrm{L}$ is lower rather than higher and that latter in age (after the age of 12 years). To model this relation age was included as a covariate in the model, in addition to the personality scores. Indeed, in this model, individuals with higher L scores were 4.28 times more likely $(1.46, \mathrm{p}<.002)$ to stay at the initial level rather than transition to a higher level as compared to individuals with lower likeability scores. This might imply that at the transition from childhood to adolescence lower likeability scores reflect a stricter and more accurate cognitive self-representation, implying more advanced transition possibilities. Figure 4 illustrates this pattern of relations from first to second testing.

Normally, the findings above imply that changes in personality are associated with the state of one's cognitive ability. To explore this model, the model above was inverted so that possible cognitive influences on personality changes would be captured. In this model, personality scores on the four dimensions at each testing wave were associated to two 
categories, staying and changing, and Gf attainment was used as the covariate to specify if personality changes across waves are affected by the initial stage of Gf. Indeed, as expected based on the results above, there was a rather small but significant effect of initial cognitive attainment on personality: those starting higher on Gf were more likely to evidence personality changes both from first to second and from first to third testing $(1.03, \mathrm{p}<.002$; odds 2.80$)$; lower personality score were associated with higher likelihood for staying unchanged from first to third testing $(-1.58, \mathrm{p} .02$; odds $=.20)$.

In conclusion, the patterns of relations described above are in line with predictions. $\mathrm{L}$ par excellence but also $\mathrm{E}$ at an earlier time do predict cognitive change and the state of cognition does predict changes in personality. Three interpretations may be given to this combination of patterns. A cognitive explanation would suggest that individuals starting lower in cognition have more room for change. Personality factors, being negatively associated with cognitive attainment, reflect, to some extent, that individuals obtaining extreme scores on personality dimensions are more likely to change cognitively because of their distance from cognitive ceiling. A personality explanation would suggest that individuals high in likeability are involved in a positive loop motivating them to advance cognitively to sustain their positive self- or social image. Individuals high in extroversion may expose themselves to learning opportunities more than introverted individuals. Finally, a moderate degree of psychoticism is related to high cognitive achievements. The studies to be presented below will hopefully further clarify these relations. A third interpretation would integrate the two interpretations above into one: changes in self-monitoring and self-regulation processes associated with the transition from rule- to principle based-thought tune cognitive functioning and self- 
presentations so that they more accurately reflect each other. The studies below will further highlight these relations.

\section{Study 2: Transition from Rule- to Principle-Based Thought and the Big Five Factors}

This study focused on the transition from rule-based to principle-based thought, involving participants from 7 through 17-year of age. These participants were examined twice by tasks addressed to processing efficiency, working memory, mathematical reasoning, and fluid intelligence. The two testing waves were separated by one year. The Croatian version of the Big Five Factors questionnaire was given at the beginning of the second testing. One of the of parents of most participants (many more mothers, 283, than fathers, 73) also answered this questionnaire for her/his child.

Study 1 above involved only fluid inferential cognitive processes and personality. This study involves, additionally, executive processes, such as attention control and working memory, and crystallized cognitive processes, such as mathematical reasoning. Thus, this study may help differentiate possible influences of personality on changes in Gf from the possible effects of executive processes. It may also highlight how personality and cognitive processes mediate parents' representations of their children. Customizing our general predictions to the present study, we would expect the following patterns of relations: First, stability factors would impede but plasticity factors would facilitate development of inferential abilities. Second, these effects would come on top of possible effects originating from executive processes, if they are specific to personality. Aspects of reasoning which involve a strong academic component which requires effort for learning, such as mathematical reasoning, would be more related to $\mathrm{C}$ than other cognitive measures. 


\section{Method}

\section{Participants}

A total of 438 right-handed participants from 7 through 17 years of age (206 male) were involved. At first testing, they were 7.92- (15, 12 males), 8.53- (68, 39 males), 9.33- (61, 34 males), 10.73- (21, 5 males), 11.39- (53, 30 males) 12.73- (38, 18 males), 13.30- (52, 28 males), 14.77- (20, 12 males), 15.41- (42, 19 males), 16.61- (35, 17 males), and 17.29-yearsold (35, 18 males), respectively. These participants were all native speakers of Croatian and lived in Zagreb, Croatia's capital. They were students in Croatian public schools and thus SES is about equally represented in each age group.

Tasks

All tests used here are described in Žebec, Demetriou, \& Kortla-Topić (2015). Specifically, the MID-KOGTESTERI was used to test speed of processing, selective and divided attention. This is a computer-based test battery that contains eight cognitive tests. For processing speed, participants responded to the appearance of stimulus (six same color Xs) by lifting their finger from a resting key and to touch the target as fast as possible. In choice reaction tasks, participants responded to one of four target stimulus by pressing the appropriate (one of four) response key. For attention control, a Stroop-based task was used: participants responded to the ink color of congruent and incongruent of color words denoting the same or a different color. The Stroop effect, which is the difference in RT between incongruent and congruent tasks is regarded as a measure of selective attention interference (MacLeod, 1991; Pashler, 1998). The divided attention (DA) task demanded simultaneous responding to two different tasks on the two panels, where the stimuli were presented in fast succession (50 to $250 \mathrm{~ms}$ ). Task 1 was a simple reaction time task from the SRT-LH test. Task 2 is an object 
size classification task from the OSC test, made in the form of two-choice RT task (Žebec, Demetriou, \& Kortla-Topić, 2015). Participants were asked to respond to Task 1 with the left hand on Panel 1, and on to Task 2 with the right hand on Panel 2. The difference between RT on Task 2 performed together with the Task 1 and RT on Task 2 performed separately was used as a measure of divided attention (Pashler, 1998). Test-retest reliability (across the two testing waves) was high, varying between .7 (CRT-C) and .85 (DA).

Working memory was addressed by the forward (FDS) and backward (BDS) digit span tasks included in the WISC-III test and extended with two items in FDS and one item in BDS (in order to increase discriminability of older age groups). Test-retest reliability across the two testing waves was satisfactory both for the FDS (.69) and the BDS (.66).

Mathematical reasoning was addressed by tasks examining the ability to execute arithmetic operations in combination to each other, algebraic reasoning, and proportional reasoning. Items in each domain scaled along four levels. In the arithmetic tasks, participants were asked to specify the operations missing from simple arithmetic equations: One (e.g., $5 *$ $3=8$ ), two (e.g., $\{4 \# 2\} * 2=6$ ), three (e.g., $\{3 * 2 \# 4\} @ 5=7$ ), and four operations (e.g., $\{5 @ 2\}$ o $4=\{12 \$ 1\} * 2$ ) were missing from the items of each level. The algebraic reasoning tasks required to specify one or more unknowns in an equation (e.g., $a+5=8$, specify $a$; $u=f+3 ; f=1$; specify $u$; if $(r=s+t)$ and $(r+s+t=30)$, specify $r$; when is true that $\{L+M+N\}=\{L+P+N\}$ ? for the four levels, respectively). In proportional reasoning, the four levels required to grasp relations between the following: (i) fully symmetrical and equivalent ratios (e.g., $1 / 2$ to 3/6); (ii) equivalent but not obviously symmetrical ratios (e.g., 2/6 to $3 / 9$ ); (iii) ordered pairs with two corresponding terms multiple of one another (e.g., $2 / 5$ to 3/7); (iv) pairs without corresponding terms (e.g., 5/12 to 3/8). In terms of the cycles of 
development specified in the introduction, the two lower levels of these batteries are primarily related to the two phases of the rule-based concepts. Levels three and four addressed the two phases of the principles cycle, respectively. This battery was found to have good psychometric and developmental properties in several studies (Demetriou \& Kyriakides, 2006). In the present sample discriminability (average index of difficulty of 35 tasks is .52 and Ferguson's $\Delta$ is .98) and reliability were high (Cronbach $\alpha=.92$, split-half $=.95$ ).

Raven's Standard Progressive Matrices involve five sets of matrices of increasing complexity. Based on Rasch scaling of performance on each of the 60 matrices, four levels were formed, each involving 15 matrices. From easy to difficult, matrices in the first group, require grasping the pattern underlying figures varying along a single dimension. In the second group, two familiar and obvious dimensions (e.g., shape, size, background, etc.) would have to be integrated. In the third group, matrices require deciphering and integrating critical dimensions through systematic search and transformation of the features involved. For instance, it is the double of ..., it goes by one more, etc. Finally, in the fourth group, matrices require deciphering multiple dimensions by grasping the thread underlying several transformations of figures and integrating into complementary general principles. Level 1 addresses abilities of the second phase of the representational cycle. Levels 2 and 3 address abilities associated with the two phases of rule-based thought, respectively. These were the levels represented in the Raven-Like test used in the study described above. Level 4 addresses abilities of first level of principle-based thought.

The translated Croatian version of the 50-item IPIP Big Five inventory included 50 items, 10 for each of the Big Five Factors. This inventory was highly reliable (Cronbach alpha $=.83)$. 
The correlations between reasoning tasks were very high (circa .5-.7). Correlations between personality measures were lower but significant (all but one .2-.3). Correlations between cognitive and personality measures varied according to personality dimension: they were positive and moderate but mostly significant in the case of $E$ (circa .2) and negative in the case of $\mathrm{C}$ (circa -.2); the rest varied between 0-.2. Child-parent correlations for the same dimension were all significant (.2-.35); the rest varied between 0 and .16 .

\section{Results}

Development. Figure 6A-C shows the developmental pattern of cognitive processes as a function of age at first and second testing wave. There were very large changes across all cognitive processes. Overall, in the cognitive domain, children progressed from modal level 1 at 7-8 years of age to modal level 3 at 16-17 years of age, $F(10,421)=136.09, \eta^{2}=.76$. Moreover, there was significant progress across all mental processes and across all age groups, $\mathrm{F}(1,427)=269.76, \eta^{2}=.39$, from first to second testing. The significant interactions between age domain, $F(30,1281)=13.96, \eta^{2}=.25$, and age and testing wave $F(10,427)=4.24, \eta^{2}=$ .09 , as well all three factors, $\mathrm{F}(30,1281)=2.66, \eta^{2}=.06$, indicated that the degree of change across age or wave differed across cognitive processes. Detailing these patterns is beyond the concerns of the present paper. However, it is of relevance to note that different domains spurted and consolidated at different age phase. It can be seen that arithmetic reasoning spurted from 7 to 11 years of age, indicating, that it is basically a rule-based acquisition;

algebraic reasoning demonstrated very little change from 7 to 11 years but developed very fast between 11 and 14 years, obviously reflecting its principle-based origins; interestingly, performance on Raven matrices developed in two spurts, one from 8 to 10, and another from 13 to 15 years, indicating that it involves a rule-based component and a principle-based 
component as expected. These patterns are informative for the cognition-personality relations to be presented below.

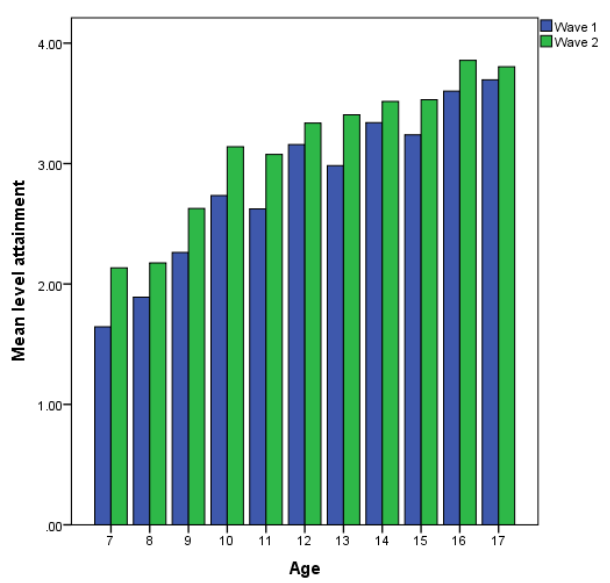

A. Arithmetic ability

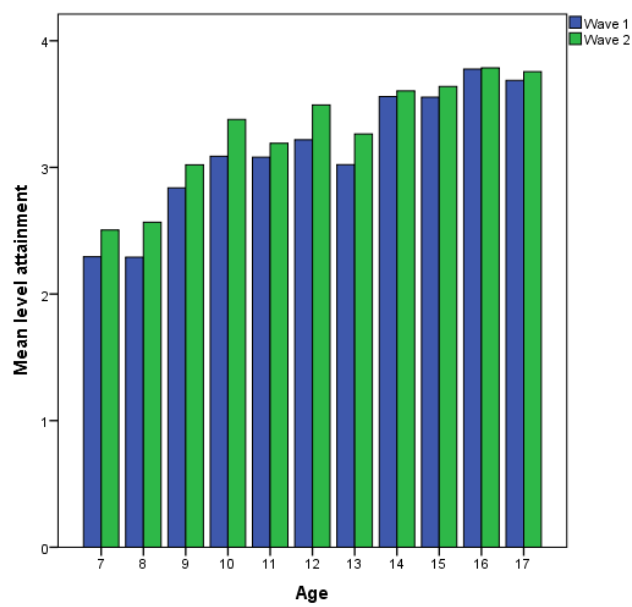

C. Raven

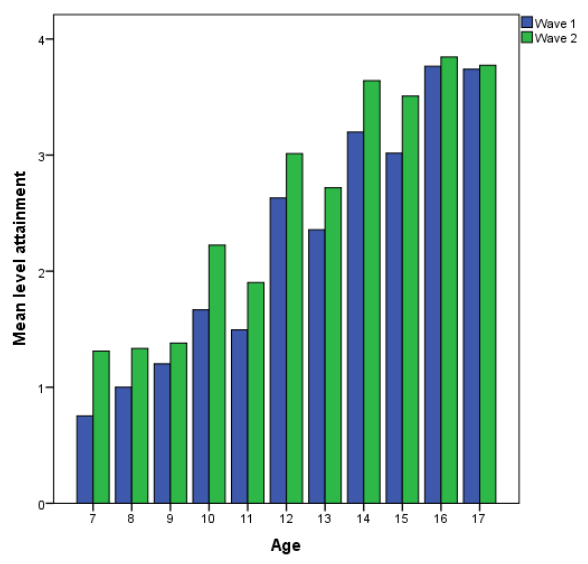

B. Algebraic reasoning

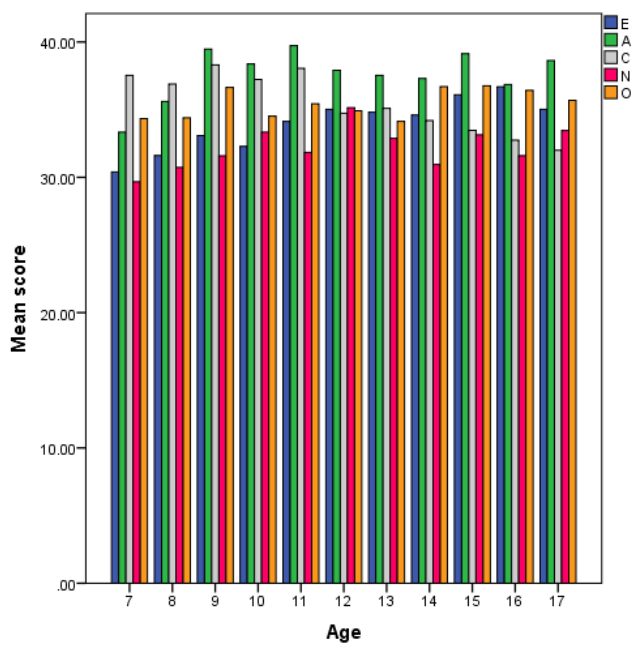

D. Personality traits

Figure 6. Mean Level Attainment in arithmetic, algebraic, and Raven reasoning

(Panels A, B, C) and personality (Panel D) as a function of age and testing wave.

In concern to personality, the main effect of age, $\mathrm{F}(10,425)=1.92, \mathrm{p}<.05$, partial $\eta^{2}=$ .04 , personality, $\mathrm{F}(4,422)=37.45, \mathrm{p}<.0001$, partial $\eta^{2}=.26$, and their interaction, $\mathrm{F}(40$, $1700)=2.78, p<.0001$, partial $\eta^{2}=.06$, were significant (see Figure 6D). Overall, scores in A 
exceeded and scores in $\mathrm{N}$ lagged behind all other factors, perhaps reflecting the two poles of social likeability, respectively. However, differences varied with age: E increased systematically from 7 to 16 years; A increased from 7 to 9 and stabilized; conscientiousness was basically steady from 7 to 10 and then decreased throughout the remaining period, resembling the inverse relation between age and $\mathrm{L}$ observed in Study 1; $\mathrm{N}$ increased from 7 to 10 and then wavered; $\mathrm{O}$ wavered throughout, with two noticeable spurts between 8 and 9 and 13 and 14. Overall, increases in personality scores occured from 7-10 years and decreases occurred in adolescence, possible reflecting the differential effect of acquiring rule-based and principle-based thought. The models below will highlight this developmental intertwining between cognitive and personality processes.

\section{Personality Mediation}

A structural equation model was first built which tested how cognitive processes from first testing wave influenced personality measures one year later and how personality measures influenced cognitive measures at second tasting. Specifically, the following first-order factors were constructed for cognitive measures at each testing wave: a processing speed factor was associated to simple reaction times; a control factor was associated to selective and divided attention; forward and backward digit span was associated to a working memory factor; arithmetic, proportionality, and algebraic reasoning were associated to a mathematical reasoning factor; Raven scores were associated to a Gf factor; the speed and attention control factors were associated to a processing efficiency factor; the working memory, mathematical reasoning, and Gf factors were associated to a factor standing for representational and inferential power (RIP). It is noted that this factor is narrower than psychometric $\mathrm{g}$ because it does not include speed and attention control measures but broader than psychometric Gf 


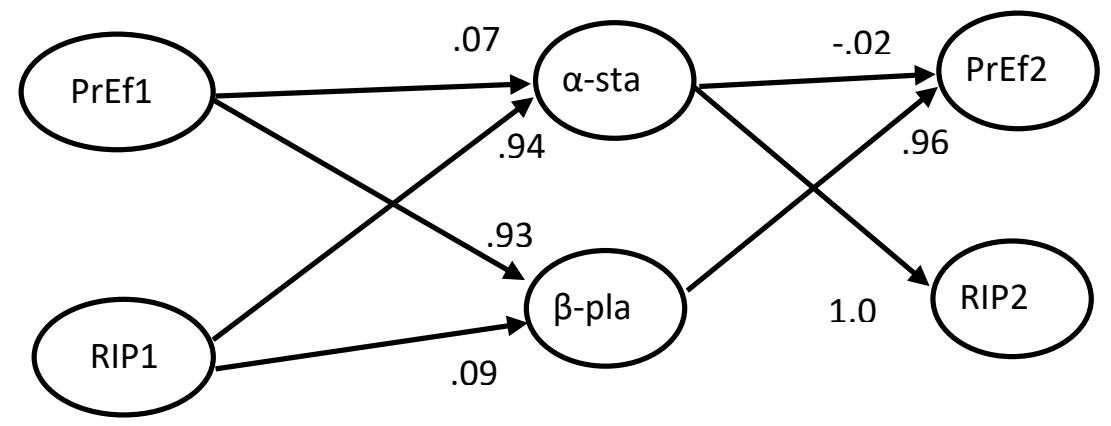

Figure 7. Cross-lagged model of the mediation of personality factors of stability ( $\alpha$-factor and plasticity ( $\beta$-factor) between processing efficiency (PrEf) and representational and inferential power (RIP) at first and second testing wave (1 and 2).

because it includes, in addition to psychometric Gf (the Raven test), working memory and mathematical reasoning. Three of the Big Five factors, i.e., A, C, and N, were associated with one factor standing for stability of personality, the $\alpha$-factor; the other two of Big Five Scores, i.e., $\mathrm{E}$ and $\mathrm{O}$, were associated with another factor standing for plasticity of personality, the $\beta$ factor. The following structural relations were built in this model. On the one hand, each of the Big Five factors was regressed on the two first wave second-order factors standing for processing and representational efficiency, to capture how first wave cognitive processes influenced personality dimensions. On the other hand, each of the two second wave cognitive factors were regressed on the two personality factors, the $\alpha$ - $\beta$-factor. Corresponding measurement errors across waves were correlated. To examine equivalence of factors across waves, the model was tested under the constraint that corresponding measurement-factor relations were free to vary or equal across testing waves.

Notably, the fit of the constrained model, $\chi^{2}(544)=1839.87, \mathrm{p}>.001, \mathrm{RMSEA}=.074$, model AIC $=751.87$, was acceptable and better than the fit of the unconstrained model, $\chi^{2}$ 
$(536)=2060.84, \mathrm{p}>.001, \mathrm{RMSEA}=.081$, model $\mathrm{AIC}=988.84$. This is the model shown in Figure 7. The pattern of relations was very interesting: the $\alpha$-factor, stability, was very strongly related to RIP (.94) and very weakly to processing efficiency (.07); plasticity, the $\beta$-factor, demonstrated the inverse pattern of relations: it was weakly related to RIP (.09) but strongly to processing efficiency (.93). This pattern of relations was replicated in the effects of personality on cognitive performance at second wave: plasticity influenced processing efficiency (.96) but not stability (-.02); stability influenced RIP (1.0). These relations were further probed in a model where personality factors were individually involved. In this model extroversion (.34), openness (.09), and agreeableness (.18) were significantly related to processing efficiency; neuroticism (.18) and conscientiousness (-.25) were related RIP. The conscientiousness-RIP negative relation replicated earlier findings about the relations between conscientiousness and intelligence.

Latent Transition analysis was again used to specify if personality is related to cognitive transition to a higher level. In this analysis level attainment on the three mathematical reasoning batteries and RPM at the two testing waves was used in the model. Specifically, level attainment at first testing on each of these cognitive dimensions was related to one category and level on each dimension at second testing was related to a second category. There were two classes in each category, those staying at the initial class and those changing, either moving to a higher class (category 1) or regressing to a lower class (category 2). At a first test of the model, attention control, working memory, and stability ( $\alpha$-factor), and flexibility ( $\beta$-factor) of personality were used as covariates. This model fit the data very well $($ Pearson Chi-Square $(199864)=4015.07, \mathrm{p}=1.0 ;$ Likelihood Ratio Chi-Square $(199864)=$ 1236.704, $\mathrm{p}=1.0 ;$ Entropy $=.88 ;$ AIC -6439.75$)$. The probability of moving to a higher level 
was good (.46) although lower than staying to the same level (.54) (odds to progress was .84). All four covariates significantly affected transition: the effect of attention control was huge $(4.85, \mathrm{p}<.001 ;$ odds $=128.14)$; the effect of working memory $(1.06 ; \mathrm{p}<.001 ;$ odds $=2.9)$ and plasticity of personality $(\beta$-factor; $\mathrm{p}<.04$; odds $=2.11)(.74)$ was significant and considerable; the effect of stability $(\alpha$-factor $)(-.76 ; \mathrm{p}<.005$; odds $=.47)$ was significant but negative. In concern to personality, these effects suggest that individuals high in flexibility were 2.11 times more likely to transition to a higher level from first to second testing that to stay at their first testing initial level. On the contrary, individuals high in stability were .47 times more likely to stay at their initial level than progress to a higher level. To further probe the origins of these effects, a second model was tested where the two personality general factors were dropped as covariates and conscientiousness and openness were used in their place. This model fit the data equally well, although slightly lower than the first model (entropy .89; AIC $=6455.66$ ). Notably, the effect of conscientiousness on transition was significant and negative $(-.70, \mathrm{p}<$ .0001 ; odds $=.57)$ and the effect of openness was significant and positive $(.96, \mathrm{p}<.04$; odds $=$ 2.61). It is strongly suggested that personality is involved in cognitive change, with some dimensions impeding and some dimensions facilitating cognitive development.

To examine if the involvement of personality in cognitive change varies with developmental phase, the LTA model above was applied separately on two age groups, 7-11year and 12-16-year-olds. Some interesting differences between these age groups were observed. First, the probability to transition to a higher state in the younger age group was limited $($ odds $=.14)$ compared to the older age group (odds $=1.13)$. This is understandable given that all reasoning domains but arithmetic were principle-based acquisitions which consolidate in adolescence rather than in childhood. Moreover, there were noticeable 
differences in the factors associated with transition in each age phase. In the younger age group, only working memory $(1.23, \mathrm{p}<.001$; odds $=3.34)$ and plasticity affected transition significantly $(1.45, \mathrm{p}<.07$; odds $=4.26)$. The pattern in the older age group was very similar to the pattern found in the total sample: attention control $(2.82, \mathrm{p}<.01$, odds $=16.76)$, working memory $(1.00, \mathrm{p}<.0001$, odds $=2.72)$, and plasticity $(.57, \mathrm{p}<.10$, odds $=1.78)$ significantly affected transition and stability exerted a negative effect $(-.72, \mathrm{p}<.05$, odds $=.49)$ started to exert a negative influence on transition. Therefore, it is suggested that based on developmental cycle, personality is differentially involved in cognitive development.

\section{Do Parents Know their Children?}

Comparing parent to children's personality self-ratings is beyond the aims of the present paper. However, we will present some findings on two dimensions, conscientiousness and extroversion. These two dimensions were shown by exploratory analysis to demonstrate significant differences between children and parents which were systematically related to cognitive development. Thus, these findings show how personality and cognition interact in influencing how parents see their children. It is noted that preliminary analysis showed no difference between ratings given by mothers and fathers. Thus, this factor was dropped from the analysis below.

Figure 8 shows the difference between children's and parents' self-ratings for conscientiousness and extroversion. It can be seen that (Panel A), from childhood to early adolescence (from 7 to 14 years), parents view their children as being less conscientious (the child-parent difference is positive) but more extrovert (the difference is negative) than children view themselves; this difference disappears in late adolescence: the main effect of personality factor, $F(1,372)=50.43, \mathrm{p}<.0001, \eta^{2}=.12$, and the interaction between this factor and age 
$\mathrm{F}(10,372)=7.72, \mathrm{p}<.0001, \eta^{2}=.17$, were highly significant. Notably, however, the pattern of differences changed significantly when two covariates were included in the analysis, performance in algebraic reasoning and attention control. Under this condition, the difference between $\mathrm{C}$ and $\mathrm{E}$ in children's and parents ratings decreased, $\mathrm{F}(1,370)=5.61, \mathrm{p}<.02, \eta^{2}=$ .02 . This change was caused by the fact these both algebra, $\mathrm{F}(1,370)=7.28, \mathrm{p}<.008, \eta^{2}=.02$, and attention control, $\mathrm{F}(1,370)=5.20, \mathrm{p}<.03, \eta^{2}=.01$, caused the child-parent difference to decrease in $\mathrm{C}$ but not in $\mathrm{E}$ (see Panel B). This is a highly interesting finding. It suggests that conscientiousness, the executive component of personality par excellence, appears tainted in the eyes of others by both a crystallized (performance in school related domain) and a fluid (attention control) aspect of the mind throughout the age span studied here. The implication of this finding will be discussed further in the discussion.

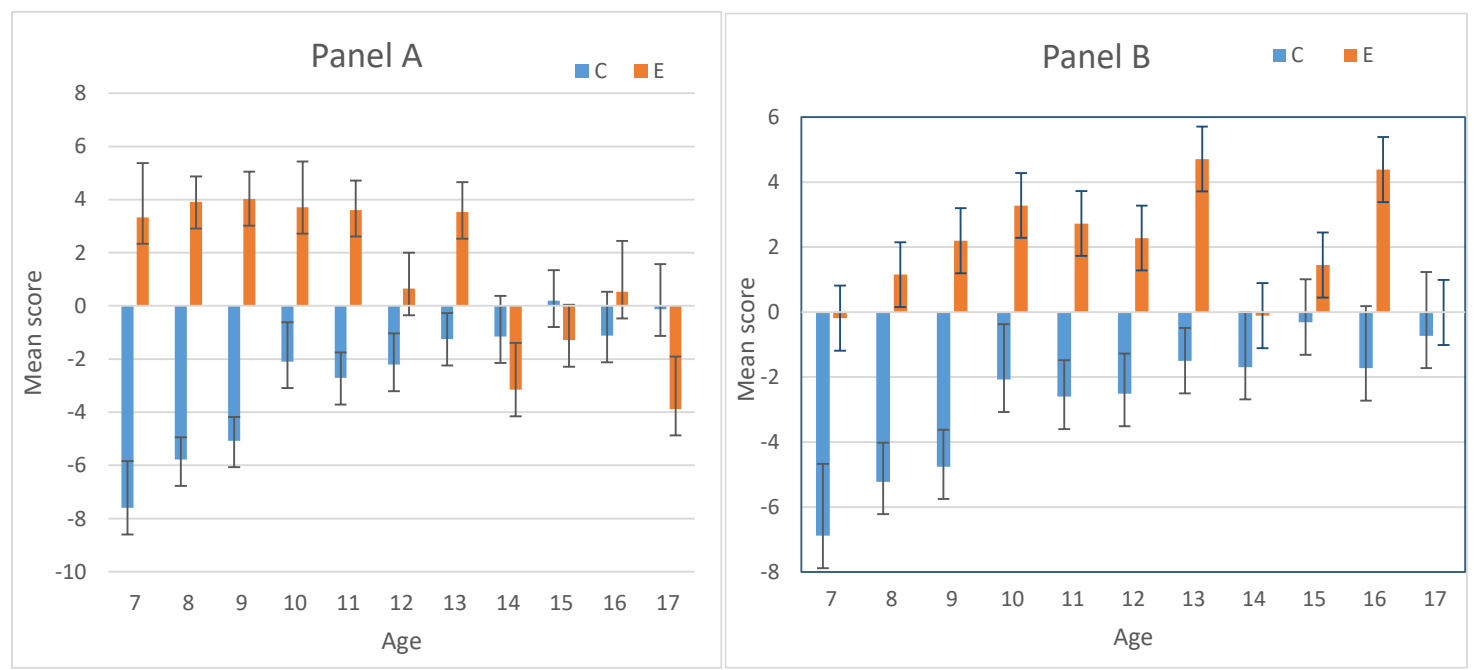

Figure 8. Differences between children's self-ratings and parents' ratings for conscientiousness (C) and extroversion (E) before (Panel A) and after (Panel B) using performance in algebraic reasoning and attention control as covariates. 


\section{Study 3: Cognition, Cognizance, Personality, Emotional Intelligence, and Academic Performance: What is the Go-between?}

This study examined a wide range of cognitive, personality, emotional intelligence, and school performance processes from 10 to 20 years age. Specifically, cognitive abilities included reasoning in several domains (inductive, quantitative, causal, spatial, and social reasoning along a range of developmental levels and cognizance (self-evaluation of own's own performance and self-representation in the domains above); personality included the Big Five Factors; emotional intelligence was examined both as a trait (self-representations about emotional characteristics) and as an ability (understanding and regulating emotions); finally, information on participants' school performance in Greek and mathematics was obtained. Therefore, this study may show how cognitive, personality, and emotional processes interact from late childhood to early adulthood and how they contribute to academic achievement. Customizing initial predictions to the present study allows testing the following: first, given the self-representational nature of personality and emotional intelligence, cognizance would occupy a central role, operating as the bridge between them; second, academic performance, being the outcome of cognitive, personality, and motivational processes, would depend on all of them.

\section{Method}

\section{Participants}

A total of 247 participants were examined. They came from fifth primary school grade $(45,25$ females; mean age $=10.7$ years $)$, first $(47,27$ females; mean age $=12.5$ years $)$, third $(42,25$ females; mean age $=14.9$ years $)$ and fifth secondary school grade $(33,22$ females; 
mean age $=16.7)$, and university $(80,55$ females; mean age 20.3 years $)$. These participants had urban residence and they were representative of the total urban population, dominated by middle-class families.

Tasks

Cognition. Cognition was examined by a part of a cognitive development test of addressed to several domains (Demetriou \& Kyriakides, 2006). For the present purposes, the following domains were involved: inductive (fluid) reasoning was addressed by six Raven-like matrices of varying complexity as specified in Study 1 (levels, ii, iii, and iv); scientific reasoning was examined by a combinatorial thinking task (specify all possible combinations in the order of drawing several differently colored balls from a bag) and hypothesis testing by properly mapping hypotheses of varying complexity with relevant patterns experiments; quantitative reasoning by algebraic (e.g., specify $\mathrm{x}$ if $\mathrm{x}=\mathrm{y}+\mathrm{z}$ and $\mathrm{x}+\mathrm{y}+\mathrm{z}=30$; when is true that $\mathrm{L}+\mathrm{M}+\mathrm{N}=\mathrm{L}+\mathrm{P}+\mathrm{N}$ ) and proportional reasoning tasks (e.g., specify how may girls and boys there are in a classroom where we have 30 children and 5 out of 6 are girls); spatial reasoning was examined by a paper folding task addressed to mental rotation and two coordination of perspectives tasks (specify the level of liquid in a tiled bottle and the direction of a an object hanging in a track moving at various inclinations); social reasoning was examined by two tasks requiring understanding of social intentions and consequences of social actions. A logit score derived from Rach-scaling all cognitive items and a mean score for each domain were used in the analyses below (Cronbach's alpha $=.88)$.

Cognizance was examined by two tests. First, participants self-evaluated their performance on a task from each of the domains included in the cognitive battery above (Raven-like matrices, hypothesis testing, algebraic reasoning, paper folding, and social 
reasoning). Each of these self-evaluation scores was standardized in relation to performance score obtained on the respective task to reflect accuracy of self-evaluation (Cronbach's alpha $=$ $.54)$.

Second, they answered a self-rating inventory involving three types of selfdescriptions: (i) domain-specific abilities related to the domains above (e.g., I can easily decipher how to solve a mathematical problem; I can easily discriminate between evidence related and not related to an event; I easily orient myself in a new city; I can grasp the hidden intentions of others); (ii) cognizance abilities related to cognition, emotions, and social behavior (e.g., I know where I am strong and where I am weak; I am able to know my body states (thoughts, emotions); I can easily shift between activities; I can appear calm when I am angry; I can focus on a task even if tired); (iii) general processing efficiency (e.g., I am very fast in learning new concepts; I can easily do calculations arithmetic calculations; I am fast in finding the solution of a problem; I can remember verbatim big chunks of text, etc.); I easily keep phone numbers in memory). Scores for self-representation in mathematical, causalscientific, spatial, and social reasoning, visual and phonological memory, self-control, and ability to know oneself were used in the analyses below (Cronbach's alpha $=.90$ ).

Personality was examined by the Greek version of the Big Five Personality Inventory. This test addressed two or three facets for each of the Big Five: achievement and order for C; anxiety and self-consciousness for $\mathrm{N}$; extroversion and introversion for E; altruism, sensitivity to others, and agreeableness for A; intellect and openness for O (Cronbach's alpha = .87).

Emotional intelligence was examined by two tests. First, trait emotional intelligence was examined a self-rating inventory (scale 1-5) addressed to knowledge about emotions (e.g., I know why my emotions change, I control my emotions, when I am in a good mood I have 
many new ideas), sensitivity in recognizing and emitting verbal and non-verbal emotional signals (e.g., I am aware of the emotional signals I send to others, I recognize someone's emotions on his phase), style of reacting to emotionally loaded events (When I get high school marks I am not affected, I am not moved by a new nice present; I am indifferent to praise), and emotional self-regulation (e.g., I control my emotions) (Cronbach's alpha $=.81$ ). This inventory was based on the emotional intelligence scale developed by Schutte et al (1998) Emotional intelligence ability was examined by two tests. The first involved three sections, each addressed to a different aspect of understanding emotions: First, participants rated the degree (1-5) of involvement of several emotions (anger, sadness, joy, disgust, fear, and surprise) in several real life episodes (obtaining high school marks, failing and wining in sports competitions, impasse in solving a problem, preparing for exams, setting goals for the school year, dealing with a dilemma). The scores given to most relevant emotion was used in each case (e.g., success-joy, failure-sorrow). Second, this test also examined the ability to specify the emotions involved in a combination of mental states, such as pleasure and expectation, joy and acceptance, sorrow and disappointment, joy and calmness, etc. Nine pairs were given which were scored on pass-fail basis. The sum of this test was used in the analysis. Third, participants were asked to write three stories about two persons shifting from one emotional state to another (e.g., from being calm and careless to being fearful and anxious; one first feels satisfied, then pleased, then enthused, and finally surprised and proud). Each story was scored on a three-point scale $(0,1$, and 2 for irrelevant, partly and fully relevant. The mean score of performance on the three stories was used in the analysis. This test is based on the test originally developed by Mayer and colleagues (Mayer, Salovey, \& Caruso, 2002; Mayer, Salovey, \& Caruso, \& Sitarenios (2003). 
To examine explicit representation of emotions, participants were asked to specify (on 1-5 scale) how much each of 15 descriptions or definitions apply to three emotions (joy, grief, and surprise). The factor score of the first principal component of a factor analysis applied on the ratings for each emotion was used in the analyses below (Cronbach's alpha $=.69$ ).

School performance was evaluated by school teachers in two subjects: Greek and mathematics. Teachers were asked to rate (on a 1-7 scale) each of their students in several domains: learning complex concepts, learning speed, originality, understanding and using complex concepts, interest, and actual performance in the subject (Cronbach's alpha $=.98$ ).

The correlations between measures varied in the fashion of the previous studies. Measures addressed to the same construct correlated moderately to high (circa 3. To .6); correlations across constructs varied between 0 to circa .3 .

\section{Results}

Developmental Patterns

Developmental patterns vary as a function of process. Expectedly, all cognitive abilities develop systematically throughout the age span covered. Individual univariate ANOVAs showed that the effect of age on (i) cognitive performance, $F(4,242)=83.70, p<$ $.0001 ; \eta^{2}=.58$, (ii) self-evaluation accuracy, $\mathrm{F}(4,242)=31.36, \mathrm{p}<.0001 ; \eta^{2}=.34$, and (iii) the understanding emotions was always very strong, $\mathrm{F}(4,242)=36.86, \mathrm{p}<.0001 ; \eta^{2}=.38$, reflecting an almost linear increase in each ability with age. Obviously, these three aspects of cognitive ability are strongly intertwined in development. Figure 9A illustrates the covariation of the product of age with the factor score of the first principal component abstracted from each of the three sets of scores. 


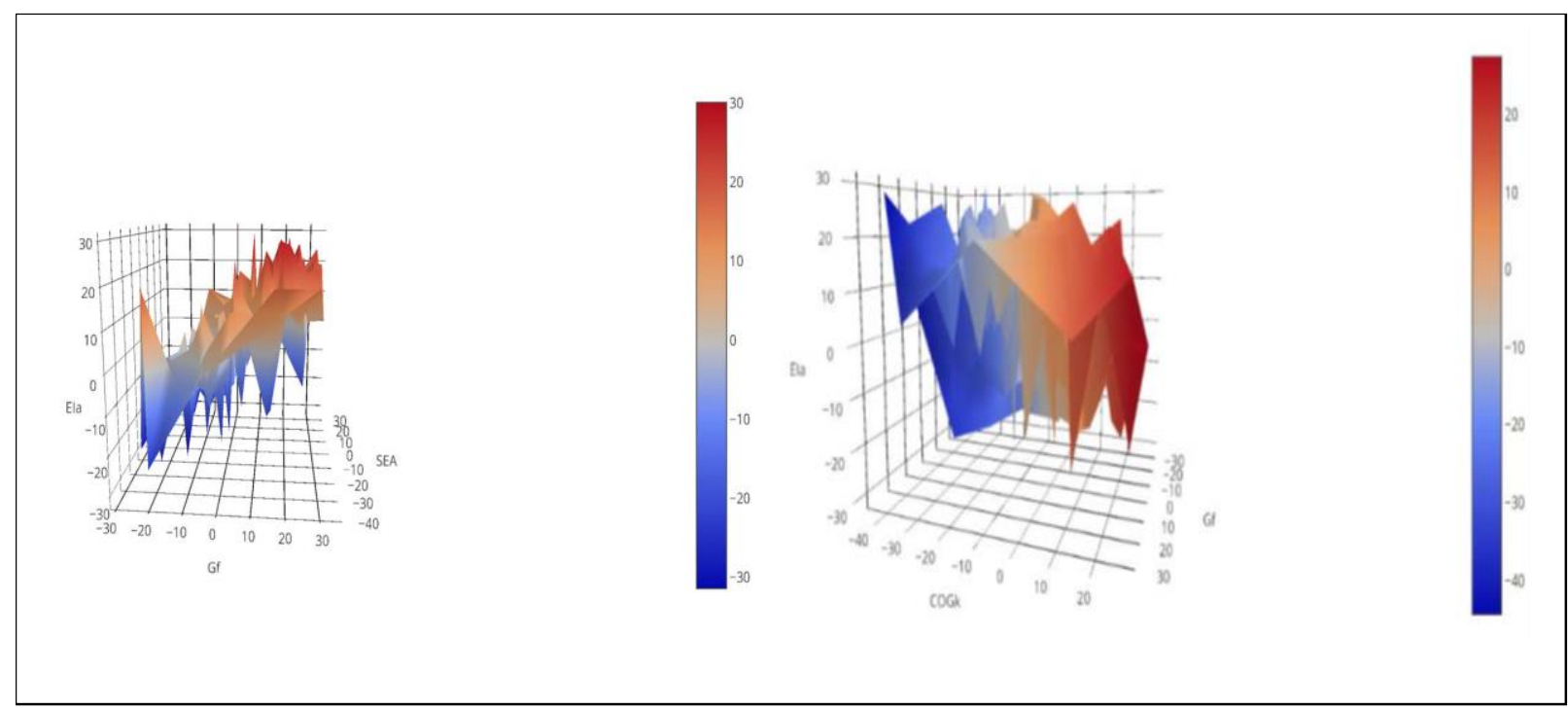

Figure 9. Three-dimensional relations between the three cognitive factors Gf, Self-evaluation accuracy (SEA) and ability to understand and define emotions (EIa) (Panel A; $\mathrm{R}^{2}$ for regressing EIa on Gf and SEA = .49) and between Gf, EIa, and self-representation of selfknowing ability (COGNsk; $\mathrm{R}^{2}$ for regressing COGNsk on Gf and EIa $=.05$ ).

Relations between age and the various traits differed from above. Specifically, selfrepresentations in cognizance (which is a second-order self-representation) decreased systematically with age. As a result, the main effect of age was non-significant, $F(4,242)=$ $.65, p>.05 ; \eta^{2}=.01$, but the interaction of age with self-representation type was highly significant, $F(4,242)=7.14, p<.0001 ; \eta^{2}=.11$. Figure 9B illustrates this negative relation between self-represented cognizance, on the one hand, and cognitive and emotional ability, on the other hand.

Similarly, the main effect of age on personality was non-significant, $F(4,242)=2.09$, $p$ $\left.>.05 ; \eta^{2}=.03\right)$. However, the main effect of personality dimension, $\mathrm{F}(4,242)=71.03, \mathrm{p}<$ 
$\left..0001 ; \eta^{2}=.54\right)$, and the age $x$ personality interaction were highly significant, $F(4,242)=$ $\left.3.61, \mathrm{p}<.0001 ; \eta^{2}=.06\right)$. These effects reflected two main trends: $\mathrm{N}$ was lower than the rest but increased with age; E was high but decreased; A decreased from 10 to 14 and increased thereafter, topping the rest after 16 years. $\mathrm{C}$ decreased from 10 to 14 and rose thereafter; $\mathrm{O}$ decreased systematically from 10 through 16 and rose from there to 20 years. In emotional intelligence, the main effect of age was again non-significant, $F(4,242)=1.21, p>.05 ; \eta^{2}=$ .02 ; however, processes did differ significantly, $\left.\mathrm{F}(2,242)=323.22, \mathrm{p}<.0001 ; \eta^{2}=.73\right)$ and they (marginally) differentially related with age, $\mathrm{F}(2,242)=1.73, \mathrm{p}<.08 ; \eta^{2}=.03$. The cognitive aspects of trait EI (understanding of emotions and regulation of emotional signals wavered with age but self-representation of emotional stability tended to decrease (Figure 10). In conclusion, expansion of cognitive ability with age is differentially reflected in various aspects of self-representation and personality with some varying with varying states of ability and some becoming stricter or more conservative. The models to be presented below will shed light on these relations.

\section{Relations between Processes}

The multiplicity of measures used in this study provides a rich basis for studying the relations between the various processes measured. Thus, two different approaches were used. First, Exploratory Graph Analysis was used to map the organization of processes. Second, structural equation modeling was used to specify their relations.

Exploratory Graph Analysis (EGA). Exploratory Graph Analysis is part of a new area called network psychometrics (see Epskamp, Maris, Waldorp \& Borsboom, 2016), which focuses on the estimation of undirected network models (i.e. Markov Random Fields; Lauritsen, 1996) to psychological datasets. EGA can show if measures form nodes that 
connected with each other into clusters standing for underlying latent variables and if nodes and clusters are related are, forming clusters standing for underlying latent variables. Golino and Demetriou (2017) showed that EGA is more accurate than other methods, including confirmatory factor analysis, to reveal the dimensions underlying performance on various cognitive test batteries under a variety of test and sample conditions. They suggested that EGA may be the method of choice to uncover underlying dimensions of behavior or ability in fields of study where clear theory specifying constructs and their relations is not yet available. Structural equation methods may then be used to validate EGA findings and more exactly specify the direction of relations between constructs.

The best-fitting EGA model, $\chi^{2}(1791)=4074.84, \mathrm{CFI}=.98, \mathrm{RMSEA}=.072$, are shown in Figure 10A. Clusters are shown different colors. It may be seen that there are three

A

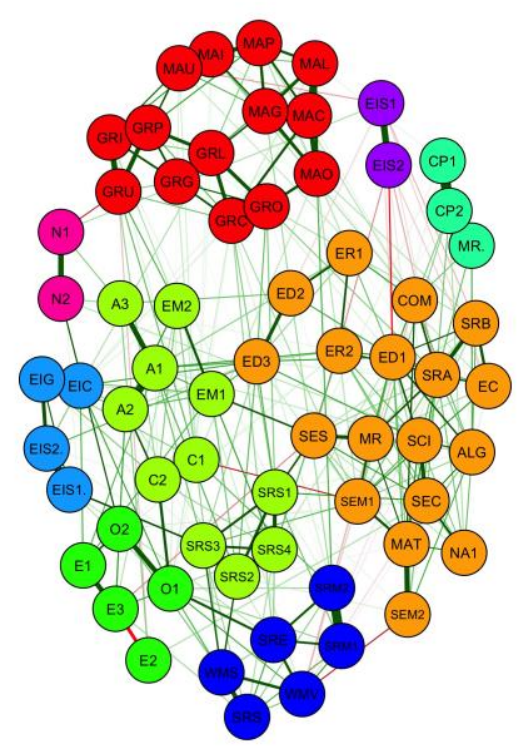

B

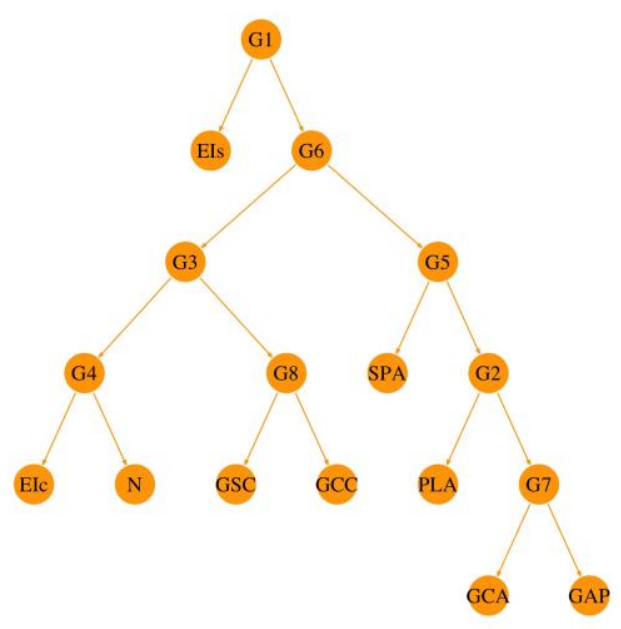


Figure 10. Network of abilities and processes as abstracted by Exploratory Graph Analysis (Panel A) and hierarchical organization of clusters as abstracted by Hierarchical Exploratory Graph Analysis.

Note: Clusters represent general academic performance measures (1: GAP), general cognitive ability (2: GCA), general self-representation of social competence cluster (3: GSC), plasticity of personality (4: PLA), spatial reasoning (5: SPA), knowing one's own emotions (6: EIc), self-representation of mental efficiency (7: GCC), emotional stability (8: EIs), and neuroticism (9: N) from the Big Five, respectively.

very broad clusters and several narrower ones. The first broad cluster included all academic performance measures (GAP). The second was a cognition cluster (GCA) including most of the cognitive and all self-evaluation accuracy (SEA) and the ability to understand emotions scores (EIa). Therefore, this is a very powerful cluster standing for general cognitive ability (GCA) as represented in Figure 9A. Notably, there was also a very broad self-representation of social competence cluster (GSC) which included self-representations of causal and social reasoning, $\mathrm{A}$ and $\mathrm{C}$ from the Big Five, and handling exchange of emotional information from EIt. Plasticity of personality, including all $\mathrm{E}$ and $\mathrm{O}$ measures, emerged as a separate cluster (PLA). Clusters 5, 6, 8, and 9 are specific, standing for spatial reasoning (5: SPA), knowing one's own emotions (6: EIc), self-representation of mental efficiency (7: GCC), emotional stability (8: EIs), and neuroticism (9: N) from the Big Five, respectively.

The hierarchical organization of these factors was explored by Hierarchical Exploratory Graph Analysis (HEGA). HEGA uses Hierarchical Random Graphs to specify the hierarchical organization of the various latent factors. In this paper we adopted a three-step approach. First, data was iteratively analyzed by EGA until all factors related to at least two items and the resulting structure was verified by CFA to fit the data well. Second, based on this structure, a 
factor score for each factor was computed for all participants; these factor scores were again fed in EGA to estimate a network of the relationships between the variables. Finally, the network obtained in the previous step was used as input to the hierarchical random graphs technique, available in the igrpah package (Clauset, Moore, \& Newman, 2008), until a consensus hierarchical network was obtained. This network is derived by weighting the hierarchical features by their likelihood based on a process akin to Bayesian model averaging. This is dendrogram model that may most likely generate the observed data (Csardi \& Nepusz, 2006).

This organization is shown in Figure 10B: it may be seen that the nine factors above are organized in two major clades, one capturing the procedural cognitive aspects of the mind $\left(\mathbf{M}_{c}\right)$ and the other the self-representational, social and emotional aspects of the mind $\left(\mathbf{M}_{s}\right)$. The $\mathbf{M}_{c}$ clade is grounded on general cognitive ability and academic performance, which integrate into a common block standing for thinking and learning (G7); this intertwines with the $\alpha$-factor, plasticity of personality to form a higher level, flexibility in cognition and learning (G2); finally, flexible cognition and learning integrate spatial cognition into a higher level (G5). $\mathbf{M}_{s}$ is grounded on two branches: the first (G8) involves self-representation of general social (GSC) and cognitive competence (GCC); the second (G4) involves $\mathrm{N}$ from the Big Five and EIc; these two branches unite into a higher level (G3) which, together with $\mathbf{M}_{c}$ (G5) form a level where the two clades merge into a higher level common (G6). This, together with emotional stability merge at the top in what Wechsler would call the personality as whole which $\mathbf{P}(\mathbf{M} c s)$.

Structural relations. To specify relations between constructs several structural equation models were compared. Only the best fitting model is presented here. This model, shown in Figure 10, involved the following first-order factors: Gf, related to performance on each of the 
cognitive domains. SEA, related to self-evaluation accuracy; $\mathrm{SR}_{\mathrm{d}}$, related to self-representation about these domains; $\mathrm{SR}_{\mathrm{sk}}$ related to self-representation of self-knowing ability; these three self-representation and self-knowing factors were related to a second-order factor standing for cognizance (COGN). There was one factor for each of the Big Five factors, associated with the facet scores included in each factor (see method); N, C, and A were related to the $\alpha$ - and E and $\mathrm{O}$ were related to the $\beta$-factor of personality; in turn, these two second-order factors were regressed on a third-order factor standing for the GFP. There were three factors capturing emotional intelligence traits (i.e., self-awareness about emotions, emotional stability, and recognition and management of emotional signals) and two factors capturing emotional intelligence as an ability (i.e., association of different emotions with corresponding real-life situation and specification of characteristics of different emotions), regressed on their corresponding second-order factors (EIt and EIa); these factors were regressed on the general emotional intelligence factor (GEI). Finally, academic performance was related to the means of performance in Greek and mathematics (GAP). The two models compared differed in the organization of these first-order factors into higher order factors. The following structural relations were built into the model: Gf was regressed on age, COGN was regressed on Gf, GFP was regressed on Gf and the residual of COGN, GEI was regressed on Gf and the residuals of COGN and GFP, and GAP was regressed on Gf and the residuals of COGN, GFP, and GFI factor. The fit of this model was very good, $\chi^{2}(1103)=1701.49, \mathrm{p}>.001, \mathrm{CFI}=.994$, RMSEA $=.058$. 


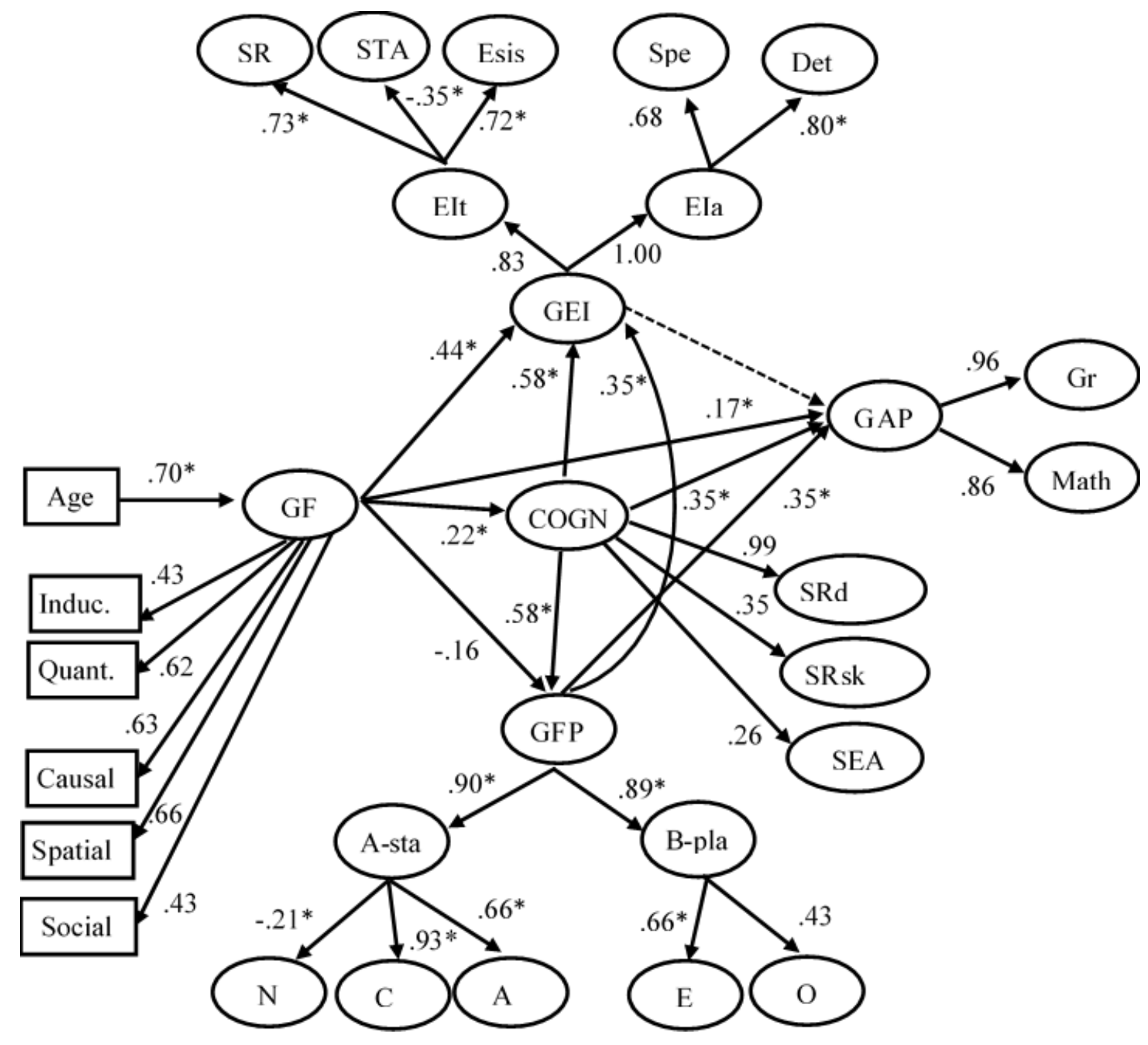

Figure 10 Figure 10. The second model of cognitive abilities, personality, emotional intelligence and school performance. $\mathrm{X}^{2}(1103)=1701.49, \mathrm{p}<.05, \mathrm{CFI}=.994, \mathrm{RMSEA}=.058(.052-.06), \mathrm{AIC}=$ 404.51 .

This model is complementary to the network and the hierarchical models presented above in that it highlights the (statistically) causal relations between the various clusters. Noticeably, cognizance was a central hub in the system: it was affected by Gf (.22) but it did affect the GFP (.58), GEI (.58) and academic performance (.35); Gf also significantly affected GEI (.44), academic performance (.17) and, non-significantly, GFP (-.17); the negative 
direction of this relation reflected the influence of A (see below); academic performance was also affected, equally by both GFP (.35) and GEI (.35).

One might note that the relation between Gf and AP was lower than is often reported in the literature. Indeed, in the present data this relation increased to .40 in a model where only the Gf and the school performance factors were involved; further, it rose to .56 when Gf and the cognizance factors were associated to a single GCA factor. Regressing cognizance on Gf (.60) and AP on Gf (.18) and the residual of cognizance (.63) showed that the later relates more strongly with AP than the former. Along the same line, a model involving only the personality and the academic performance factors indicated that personality effects on school performance, in addition to the GFP as such (.26), originated from C (.33), A (-.19) and O $(.15)$.

This model was tested in a two-group analysis including participants from 10 to 14 years in one group and participants from 16 to 20 years in another group. All measurementfactor relations were constrained to be equal across the two groups; the factor-factor relations were left free to vary across the groups to examine if relations between factors change with age. The fit of this model was good, $\chi^{2}(2003)=3231.85, \mathrm{p}>.001, \mathrm{RMSEA}=.071$, model AIC $=-774.35$. Of the various relations, only three noticeable differences between these two age groups emerged: on the one hand, the effect of Gf on the GEI in the younger group (.33) was significantly higher $(\mathrm{z}=3.22, \mathrm{p}<.01)$ than in the older group $(-.10)$; on the other hand, the effect of Gf on cognizance (.19 vs. .29 for the younger and older participants, respectively; $\mathrm{z}=$ $1.06, \mathrm{p}>.05)$ and the effect of cognizance on the GFP (.55 vs. .93; $\mathrm{z}=2.41, \mathrm{p}<.01)$ were larger in the older group. This pattern of age differences indicates that early in adolescence, a period of transition from rule- to principle-based thought, cognitive changes influence EI; 
later, when cognitive ability gets stabilized and cognizance becomes sharper and more accurate, self-representations and self-characterizations in personality also become more accurate, and some-times stricter than early in development. As discussed below, these relations are generally in line with predictions.

\section{Conclusions}

The findings of the three studies presented here suggest a clear conclusion about the state of the constructs examined: Cognition and personality are distinct but related, forming two converging clades which organize various more specific processes in each. We trust that the great scholars of the field mentioned in the introduction, Allport, Wechsler, and Jensen, would be pleased to see how cognition $(\mathbf{M} c)$ and personality $(\mathbf{M} s)$ merge into $\mathbf{P}(\mathbf{M} c s)$ in Figure 10B. The network model suggested by EGA and the hierarchical model suggested by HEGA suggest that some processes in each clade interlock this clade with the other. Mental plasticity (E and $\mathrm{O}$ ) is personality's envoy in cognition; cognizance is cognition's envoy in personality. The so called emotional intelligence does not seem to be an autonomous dimension of the person. Its cognitive components are completely absorbed by the inferential clade and its social components appear $\mathbf{M} s$ highlighting the peculiarities of the social interactive aspects of personality. These findings fully confirm recent research showing that emotional intelligence is probably synonymous with the processes captured by the GFP (van der Linden, Pekaar, Schrmer, Vernon, Dunkel, \& Petrides, 2017).

Relations between cognition and personality do vary with development. According to our first prediction, self-representations improve with age and liaise between cognition and personality with increasing accuracy and refinement. Study 3 explicitly showed that the accuracy of self-evaluations of actual cognitive performance improved systematically from 9 
to 20 years along with improvements in sheer cognitive ability. In fact, alignment of all aspects of self-representation with each other and cognitive ability tightened with age, suggesting that cognitive processing, be it object- or socially-emotionally oriented, becomes inseparable from the various aspects of cognizance.

According to the second prediction, cognizance would function as the central hub between all other functions involved. This was indeed the case, as clearly suggested by Study 3. In fact, this role expressed itself in several seemingly unrelated trends. Specifically, at a first glance, it would be paradoxical that Eysenck's social likeability would appear as a very powerful developmental factor, diminishing systematically with age and cognitive development. Obviously, L stands up, among the other personality factors, as a strong selfrepresentational agent. Hence, its similar developmental trends with self-representation of one's knowing ability mapped by Study 3. Obviously, decrease here does not imply loss of ability; it implies adjustments in self-representations of both cognitive and social characteristics, along with cognitive improvement. These seemingly contrasting trends give a developmental dimension to Socrates epistemic insight: "The only thing I know is that I know nothing” is not just an attainment of great philosophical minds. It is a developmental construction inherent in cognitive change which is gradually integrated into the development of personality.

According to the third prediction, some aspects of personality have a privileged relation with various aspects of cognition. This was a standard finding across all three studies. On the one hand, both dimensions of plasticity, the $\alpha$-factor, proved to be integral components of the mind. It is reminded that in Study $1 \mathrm{E}$ was related to the state of Gf two years later. In Study 2 , the $\alpha$-factor as such, $\mathrm{O}$ in particular, contributed to the likelihood of longitudinal 
change, especially in adolescence. Notably, the inverse relation was also observed. That is, cognitive changes did raise the likelihood for changes in personality. In Study 3, the $\alpha$-factor aligned with the cognitive rather than with the personality clade of whole person. On the other hand, some aspects of the $\beta$-factor, $\mathrm{C}$ in particular, were negatively related to the likelihood of transition across cognitive developmental levels (Study 2). In contrast, in line with the expected paradox, $\mathrm{C}$ did relate positively with academic performance. One might assume that this apparent paradox simply reflects the double role of $\mathrm{C}$ in actual functioning in real life. The first: individuals high in $\mathrm{C}$ are slower in cognitive change perhaps settling lower at their final cognitive level because they are conservative in facing challenges inducing change; this is the advantage of individuals high in $\mathrm{O}$ and $\mathrm{E}$. The second: this very seeming weakness provides an advantage in realms of activity where self-discipline, order, and systematicity matter in the long run. Obviously, school learning and academic performance is one such realm, par excellence.

Developmental phase is crucial in cognition-personality interactions. In late childhood, the consolidation of rule-based thought and the turn of cognizance to internal mental and personality processes causes an inflation and overgeneralization of self-value judgements, reflecting this developmentally nascent sense of mental and behavioral power. Later in adolescence, emergence of principle-based thought and increasing resolution of self-knowing causes a more conservative approach to self-representation which integrates epistemic recognition of the limits of one's own mental power with the limitations of one's positive characteristics.

\section{References}

Ackerman, P. L. (2018). The search for personality-intelligence relations: Methodological and 
conceptual issues. Journal of Intelligence, 6(1), 2, doi:10.3390/jintelligence6010002

Allport, G. W. (1937). Personality: A psychological interpretation. New York: Henry Holt.

Antinori, A., Carter, O. L., \& Smillie, L. D. (2017). Seeing it both ways: Openness to experience and binocular rivalry suppression. Journal of Research in Personality, 68, 15-22.

Asendorpf, J. B., \& van Aken, M. A. G. (2003). Validity of Big Five Personality Judgments in childhood: A 9 year longitudinal study. European Journal of Personality, 17, 1-17.

Barrett, P.T., Petrides, K.V., Eysenck S.B.G., \& Eysenck H.J. (1998). "The Eysenck Personality Questionnaire: an examination of the factorial similarity of P, E, N, and L across 34 countries". Personality and Individual Differences, 25, 805-819.

Blair, C. (2006). How similar are fluid cognition and general intelligence? A developmental neuroscience perspective on fluid cognition as an aspect of human cognitive ability. Behavioral and Brain Sciences, 29,109-160.

Carroll, J. B. (1993). Human cognitive abilities: A survey of factor-analytic studies; New York: Cambridge University Press.

Case, R. (1992). The mind's staircase: Exploring the conceptual underpinnings of children's thought and knowledge. Hillsdale, NJ: Erlbaum.

Caspi, A. (2005). Personality development: Stability and change. Annual Review of Psychology, $56,453-484$.

Cattell, R.B. (1965). The Scientific Analysis of Personality. NYC, NY: Penguin Group.

Chamorro-Premuzic, T., \& Furnham, A. (2006). Personality and intellectual competence. Mahwah, NJ: Lawrence Erlbaum.

Clauset, A., Moore, C., \& Newman, M. E. (2008). Hierarchical structure and the prediction of missing links in networks. Nature, 453(7191), 98-101. 
Costa, P. T, Jr., \& McCrae, R. R. (1992). Four ways five factors are basic. Personality and Individual Differences, 13, 653-665.

Csardi G. \& Nepusz, T. (2006). The igraph software package for complex network research, InterJournal, Complex Systems 1695. 2006. http://igraph.org.

Demetriou, A., (2000). Organization and development of self-understanding and self- regulation: Toward a general theory. In M. Boekaerts, P.R. Pintrich, \& M. Zeidner (Eds.), Handbook of self-regulation (pp. 209-251). Academic Press.

Demetriou, A., Christou, C., Spanoudis, G., \& Platsidou, M. (2002). The development of mental processing: Efficiency, working memory, and thinking. Monographs of the Society of Research in Child Development, 67, Serial Number 268.

Demetriou, A., \& Kyriakides, L. (2006). A Rasch-measurement model analysis of cognitive developmental sequences: Validating a comprehensive theory of cognitive development. British Journal of Educational Psychology, 76, 209-242.

Demetriou, A., Kyriakides, L., \& Avraamidou, C. (2003). The Missing link in the relations between intelligence and personality. Journal of Research in Personality, 37, $547-581$.

Demetriou, A., Makris, N., Kazi, S., Spanoudis, G., \& Shayer, M. (2018a). The developmental trinity of mind: Cognizance, executive control, and reasoning. WIREs Cognitive Science, https://doi.org/10.1002/wcs.1461

Demetriou, A., Makris, N., Kazi, S., Spanoudis, G., Shayer, M., \& Kazali, E. (2018b). Mapping the dimensions of general intelligence: An integrated differentialdevelopmental theory. Human Development, 61, 4-41.

Demetriou, A., \& Spanoudis, G. (2018). Growing minds: A developmental theory of 
intelligence, brain and education. London: Routledge.

Demetriou, A., Spanoudis, G., Kazi, S., Mouyi, A., Žebec., M. S., Kazali, E., Golino, H. F., Bakracevic, K., \& Shayer (2017). Developmental differentiation and binding of mental processes with re-morphing g through the life-span. Journal of Intelligence, 5, 23; doi:10.3390/jintelligence5020023.

Epskamp, S., Maris, G., Waldorp, L. J., \& Borsboom, D. (2016). Network psychometrics. arXiv:1609.02818.

Eysenck, H. J., Eysenck, S. B. G. (1969) The structure and measurement of personality. London: Routledge \& Paul.

Eysenck, H. J. (1992a). A reply to Costa and McCrae. P or A and C-the role of theory. Personality and Individual Differences, 13, 867-868.

Eysenck, H. J. (1992b). Four ways five factors are not basic. Personality and Individual Differences, 13, 667-67.

Flynn, J. R. (2009). What is intelligence: Beyond the Flynn effect. Cambridge: Cambridge University Press.

Golino, H., F. \& Demetriou, A. (2017). Estimating the dimensionality of intelligence like data using Exploratory Graph Analysis. Intelligence, 62, 54-70.

Hunt, E. (2011). Human Intelligence, Cambridge: Cambridge University Press.

Jensen, A. R (1998). The g factor: The science of mental ability. Westport, CT: Praeger.

Lamb, M. E., Chuang, S. S., Wessels, H., Broberg, A. G., Hwang, C. P. (2002). Emergence and construct validation of the Big Five Factors in early childhood: A longitudinal analysis of their ontogeny in Sweden. Child Development, 73, 1517-1524.

MacDonal, K. B. (2008). Effortful control, explicit processing, and the regulation of human 
evolved predispositions. Psychological Review, 115, 1012-1031.

Mayer, J. D., Salovey, P., \& Caruso, D. R. (2002). MSCEIT user's manual. Toronto: MultiHealth Systems.

Mayer, J. D., Salovey, P., \& Caruso, D. R., \& Sitarenios, G. (2003). Measuring emotional intelligence with the MSCEIT V2.0. Emotion, 3, 97-105.

Mayer, J. D., Salovey, P., \& Caruso, D. R. (2004). Emotional intelligence: Theory, Findings and implications. Psychological Inguiry, 15, 197-215.

McIntyre, M., \& Graziano, W. G. (2016). Seeing people, seeing things: Individual differences in delective attention. Personality and Social Psychology Bulletin, 1-14, DOI: $10.1177 / 0146167216653937$.

Muthen, B., \& Asparouhov, T. (2011). LTA in Mplus: Transition probabilities inuenced by covariates. Mplus Web Notes: No. 13

Pascual-Leone, J. (1987). Organismic processes for neo-Piagetian theories: A dialectical causal account of cognitive development. International Journal of Psychology, 22, $531-570$.

Pashler, H.E. (1998). The Psychology of attention. Massachussetts: A Beadford Book (MIT Press).

Piaget, J. (1970). Piaget's theory. In P. H. Mussen (Ed.), Carmichael's handbook of child development (pp. 703-732). New York, NY: Wiley.

Roberts, B. W., Walton, K. E., \& Viechtbauer, W. (2006). Patterns of mean-level change in personality traits across the life course: A meta-analysis of longitudinal studies. Psychological Bulletin, 132, 1-15. 
Rothbart, M. K. (2011). Becoming who we are: Temperament and personality in development. New York. Guilford.

Rushton, J. P., \& Irwing, P. (2009). A General Factor of Personality in 16 sets of the Big Five, the Guilford-Zimmerman Temperament Survey, the California Psychological Inventory, and the Temperament and Character Inventory. Personality and Individual Differences, $47,558-564$.

Shayer, M., Demetriou, A., \& Pervez, M. (1988). The structure and scaling of concrete operational thought: Three studies in four countries. Genetic, Social and General Psychology Monographs, 114, 307-376.

Schutte, N. S., Malouff, J. M., Hall, L. E., Haggerty, D. J., Cooper, J. T., Golden, C. J., \& Dornheim, L. (1998). Development and validation of a measure of emotional intelligence. Personality and Individual Differences, 25, 167-177.

van der Linden, D., Pekaar, K. A., Schrmer, J. A., Vernon, P. A. Dunkel, C. S. \& Petrides, K. V. (2017). Overlap between the General Factor of Personality and Emotional Intelligence: A Meta-Analysis. Psychological Bulletin, 143, 36-52.

van der Linden, D., te Nijenhuis, I., \& Bakker, A. B. (2010). The General Factor of Personality: A meta-analysis of Big Five intercorrelations and a criterion-related validity study. Journal of Research in Personality, 44, 315-327.

Wechsler, D. (1935). The range of human abilities. Baltimore, MD: William \& Wilkins.

Zebec, M., Demetriou, a., \& Topic, M. (2015). Changing expressions of general intelligence in development: A 2-wave longitudinal study from 7 to 18 Years of age. Intelligence, 49, 94-109. 
Ziegler, M., Danay, E., Heene, M., Asendorpf, J., \& Buhner, M. (2012). Openness, fluid intelligence, and crystallized intelligence: Toward an integrative model. Journal of Research in Personality, 46, 173-183.

Zuckerman, M., Kuhlman, D. M., Joireman, J., Teta, P., \& Kraft, M. (1993). A comparison of three structural models for personality: The Big Three, the Big Five, and the Alternative Five. Journal of Personality and Social Psychology, 65, 757-768. 